\title{
Dilepton Spectroscopy of QCD Matter at Collider Energies
}

\author{
Ralf Rapp \\ Cyclotron Institute and Department of Physics \& Astronomy, Texas A\&M University, College Station, TX 77843-3366, USA
}

Correspondence should be addressed to Ralf Rapp; rapp@comp.tamu.edu

Received 3 April 2013; Accepted 4 July 2013

Academic Editor: Edward Sarkisyan-Grinbaum

Copyright (C) 2013 Ralf Rapp. This is an open access article distributed under the Creative Commons Attribution License, which permits unrestricted use, distribution, and reproduction in any medium, provided the original work is properly cited.

\begin{abstract}
Low-mass dilepton spectra as measured in high-energy heavy-ion collisions are a unique tool to obtain spectroscopic information about the strongly interacting medium produced in these reactions. Specifically, in-medium modifications of the vector spectral function, which is well known in the vacuum, can be deduced from the thermal radiation off the expanding QCD fireball. This, in particular, allows to investigate the fate of the $\rho$ resonance in the dense medium and possibly infer from it signatures of the (partial) restoration of chiral symmetry, which is spontaneously broken in the QCD vacuum. After briefly reviewing calculations of thermal dilepton emission rates from hot QCD matter, utilizing effective hadronic theory, lattice QCD, or resummed perturbative QCD, we focus on applications to dilepton spectra at heavy-ion collider experiments at RHIC and LHC. This includes invariantmass spectra at full RHIC energy with transverse-momentum dependencies and azimuthal asymmetries, as well as a systematic investigation of the excitation function down to fixed-target energies, thus making contact to previous precision measurements at the SPS. Furthermore, predictions for the energy frontier at the LHC are presented in both dielectron and dimuon channels.
\end{abstract}

\section{Introduction}

The exploration of matter at extremes of temperature $(T)$ and baryon density $\left(\rho_{B}\right)$ is at the forefront of research in contemporary nuclear physics, with intimate connections to high-energy, condensed-matter, and even atomic physics [1]. Theoretical efforts over the last few decades are suggesting an extraordinary richness of the phase diagram of strongly interacting matter, which should ultimately emerge from the underlying theory of quantum chromodynamics (QCD) as part of the standard model. However, several basic questions, both qualitative and quantitative, such as the possible existence of first order transitions and their location as function of baryon-chemical potential $\left(\mu_{B}\right)$ and temperature, remain open to date [2]. A close interplay of experiment and theory is needed to create a robust knowledge about the QCD phase structure. On one hand, naturally occurring matter at temperatures close to or beyond the expected pseudo-critical one, $T_{\mathrm{pc}} \simeq 160 \mathrm{MeV}[3,4]$, may last have existed $\sim 14$ billion years ago, during the first tens of microseconds of the Universe. On the other hand, at small temperatures, matter with baryon densities close to or beyond the critical one for the transition into quark matter may prevail in the interior of compact stars today, but its verification and exploration from observational data are challenging [5]. It is quite fascinating that tiny manmade samples of hot QCD matter can nowadays be created and studied in the laboratory using ultrarelativistic heavy-ion collisions (URHICs). Significant progress has been made in understanding the properties of this medium through analyses of experiments conducted at the CERN's Super-Proton Synchrotron (SPS), BNL's Relativistic Heavy-Ion Collider (RHIC), and CERN's Large Hadron Collider (LHC) (see, e.g., the recent Quark Matter conference proceedings $[6,7])$. For example, systematic investigations of the produced hadron spectra have revealed a hydrodynamic behavior of the bulk matter in the region of low transverse momenta $\left(q_{t} \lesssim\right.$ 2-3 GeV) and a strong absorption of hadrons with high transverse momentum $\left(q_{t} \gtrsim 6 \mathrm{GeV}\right)$. Even hadrons containing a heavy quark (charm or bottom) exhibit substantial energy loss and collectivity due to their coupling to the expanding fireball. While the total charm and bottom yields are essentially conserved, the production of heavy quark-antiquark bound states (charmonia and bottomonia) is largely suppressed. The relation of the above hadronic observables to spectral properties of the medium is, however, rather indirect. Low-mass dileptons, on the other hand, are radiated from the interior of the medium throughout the fireball's lifetime, as 
their mean-free path is much larger than the size of the fireball. Thus, their invariant-mass spectra directly measure the in-medium vector spectral function, albeit in a superposition of the varying temperature in the fireball's expansion.

The dilepton program at the SPS has produced remarkable results. The CERES/NA45 dielectron data in $\mathrm{Pb}-\mathrm{Au}$ collisions, and particularly the NA60 dimuon spectra in In-In collisions, have shown that the $\rho$-meson undergoes a strong broadening, even complete melting, of its resonance structure, with quantitative sensitivity to its spectral shape, see [8-10] for recent reviews. The QCD medium at SPS energies is characterized by a significant net-baryon content with chemical potentials of $\mu_{B} \simeq 250 \mathrm{MeV}$ at chemical freezeout, $T_{\mathrm{ch}} \simeq 160 \mathrm{MeV}$ [11], and further increasing as the system cools down [12]. Baryons have been identified as a dominant contributor to the medium modifications of the $\rho$ 's spectral function [10]. The question arises how these develop when moving toward the net baryon-free regime in the QCD phase diagram, $\mu_{B} \ll T$. Theoretical expectations based on the hadronic many-body approach [13] suggest comparable medium effects in this regime, since the relevant quantity is the sum of baryon and antibaryon densities, and this turns out to be similar at SPS and RHIC/LHC [12], at least close to $T_{\mathrm{pc}}$. Since $T_{\mathrm{ch}} \simeq T_{\mathrm{pc}}$ at collider energies, the total baryon density at RHIC and LHC in the subsequent hadronic evolution of the fireball will remain similar. We also note that the $\mu_{B} \simeq 0 \mathrm{MeV}$ regime is amenable to numerical lattice QCD calculations, both for the equation of state of the medium evolution, and in particular for the microscopic dilepton production rate, at least in the QGP phase for now [14, 15]. Furthermore, since the phase transition at $\mu_{B} \simeq 0 \mathrm{MeV}$ presumably is a continuous crossover [16], a realistic dilepton rate should vary smoothly when changing the temperature through $T_{\mathrm{pc}}$. Thus, after the successful fixed-target dilepton program at the CERN-SPS, the efforts and attention are now shifting to collider energies around experiments at RHIC and LHC.

In the present paper we will focus on the theory and phenomenology of dilepton production at collider energies (for a recent overview including an assessment of SPS data, see, e.g., [17]). The presented material is partly of review nature, but also contains thus far unpublished results, for example, updates in the use of nonperturbative QGP dilepton rates and equation of state, and detailed predictions for invariantmass and transverse-momentum spectra for ongoing and upcoming experiments at RHIC and LHC, including an excitation function of the beam energy scan program at RHIC.

This paper is organized as follows. In Section 2, we briefly review the calculation of the thermal dilepton emission rates from hadronic matter and the quark-gluon plasma (QGP). We elaborate on how recent lattice-QCD results at vanishing three-momentum $(q=0)$ may be extended to finite $q$ to enable their application to URHICs. In Section 3, we discuss in some detail the calculations of dilepton spectra suitable for comparison with experiment; this involves a brief discussion of the medium evolution in URHICs (including an update of the equation of state) in Section 3.1 and of nonthermal sources (primordial production and final-state decays) in Section 3.2. It will be followed by analyses of mass and momentum spectra, as well as elliptic flow at full
RHIC energy in Section 3.3, and of an excitation function as obtained from the RHIC beam energy scan in Section 3.4; predictions for dielectron and dimuon spectra at current (2.76 ATeV) and future (5.5 ATeV) LHC energies are presented in Section 3.5. We end with a summary and outlook in Section 4.

\section{Thermal Dilepton Rates in QCD Matter}

The basic quantity for connecting calculations of the electromagnetic (EM) spectral function in QCD matter to measurements of dileptons in heavy-ion collisions is their thermal emission rate; per unit phase space, it can be written as

$$
\frac{d N_{l l}}{d^{4} x d^{4} q}=-\frac{\alpha_{\mathrm{EM}}^{2} L(M)}{\pi^{3} M^{2}} f^{B}\left(q_{0} ; T\right) \operatorname{Im} \Pi_{\mathrm{EM}}\left(M, q ; \mu_{B}, T\right),
$$

where $L(M)$ is a lepton phase-space factor (=1 for vanishing lepton mass), $f^{B}$ denotes the thermal Bose distribution, and $q_{0}=\sqrt{M^{2}+q^{2}}$ is the energy of the lepton pair (or virtual photon) in terms of its invariant mass and 3-momentum. As mentioned above, this observable is unique in its direct access to an in-medium spectral function of the formed system, namely, in the vector (or EM) channel, $\operatorname{Im} \Pi_{\mathrm{EM}} \equiv$ $(1 / 3) g_{\mu \nu} \operatorname{Im} \Pi_{\mathrm{EM}}^{\mu \nu}$. It is defined via the correlation function of the EM current, $j_{\mathrm{EM}}^{\mu}$, as transported by the electric-charge carriers in the system. In quark basis, the EM current is given by the charge-weighted sum over flavor:

$$
j_{\mathrm{EM}}^{\mu}=\sum_{q=u, d, s} e_{q} \bar{q} \gamma^{\mu} q,
$$

while in hadronic basis, it is in good approximation given by the vector-meson fields:

$$
j_{\mathrm{EM}}^{\mu}=\sum_{V=\rho, \omega, \phi} \frac{m_{V}^{2}}{g_{V}} V^{\mu},
$$

known as vector-dominance model (VDM). Since the significance of thermal dilepton radiation is limited to masses below the $J / \psi$ mass, $M \lesssim 3 \mathrm{GeV}$, we will focus on the light- and strange-quark sector in this article.

In the vacuum, the EM spectral function is well known from the $e^{+} e^{-}$annihilation cross section into hadrons, usually quoted relative to the annihilation into dimuons as the ratio $R=-(12 \pi / s) \operatorname{Im} \Pi_{\mathrm{EM}}(\mathrm{cf}$. Figure 1). It illustrates that the nonperturbative hadronic description in terms of VDM works well in the low-mass region (LMR), $M \lesssim 1 \mathrm{GeV}$, while the perturbative partonic description appears to apply for $M \gtrsim$ $1.5 \mathrm{GeV}$. Thus, in URHICs, dilepton spectra in the LMR are ideally suited to study the properties of vector mesons in the medium. A central question is if and how these medium modifications can signal (the approach to) deconfinement and the restoration of the dynamical breaking of chiral symmetry (DBCS). After all, confinement and DBCS govern the properties of hadrons in vacuum. At masses $M \gtrsim 1.5 \mathrm{GeV}$, the perturbative nature of the EM spectral function suggests that in-medium modifications are suppressed, coming in as 


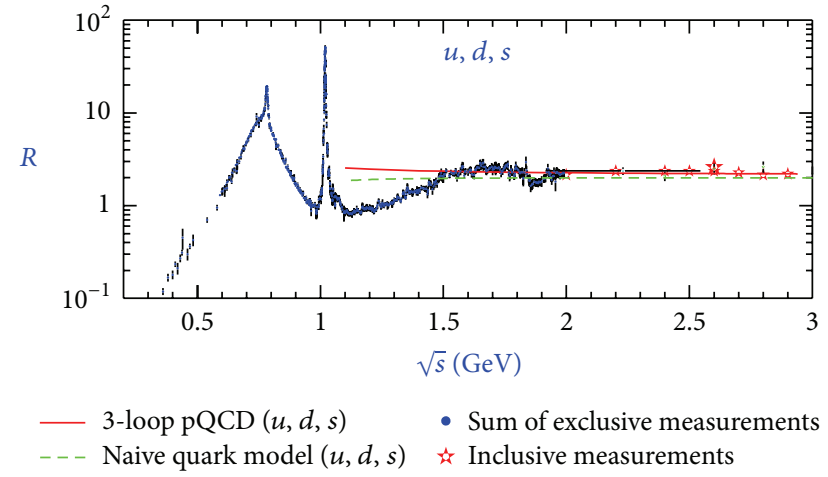

FIgURE 1: Compilation of experimental data for the ratio, $R$, of cross sections for $e^{+} e^{-} \rightarrow$ hadrons over $e^{+} e^{-} \rightarrow \mu^{+} \mu^{-}$, as a function of invariant mass $\sqrt{s}=M$. Figure taken from [33].

corrections in powers of $T / M$ and $\alpha_{s}$. In this case, invariantmass spectra of thermal radiation become an excellent measure for the prevalent temperatures of the produced system, free from blue shifts due to the medium expansion which strongly affect $p_{t}$ spectra.

2.1. Hadronic Matter. Over the last two decades, broad efforts have been undertaken to evaluate the medium modifications of the $\rho$-meson. The latter dominates in the EM spectral function over the $\omega$ by about a factor of 10 (the $\phi$ appears to be rather protected from hadronic medium effects, presumably due to the OZI rule, at least for its coupling to baryons). Recent overviews of these efforts can be found, for example, in $[10,18,19]$. Most approaches utilize effective hadronic (chiral) Lagrangians and apply them in diagrammatic many-body theory to compute thermal (or density) loop corrections. The generic outcome is that of a substantial broadening of the $\rho$ 's spectral shape, with little mass shift (in a heat bath, chiral symmetry protects the $\rho$ from mass shifts at order $\mathcal{O}\left(T^{2}\right)$ [20]). The magnitude of the $\rho$ 's in-medium width (and/or its precise spectral shape) varies in different calculations, but the discrepancies can be mostly traced back to the differing contributions accounted for in the Lagrangian (e.g., the set of baryon and/or meson resonance excitations, or medium effects in the $\rho$ 's pion cloud). Similar findings arise when utilizing empirically extracted on-shell $\rho$-meson scattering amplitudes off hadrons in linear-density approximation [21]. Since these calculations are restricted to resonances above the nominal $\rho N$ (or $\rho \pi$ ) threshold, quantitative differences to many-body (field-theoretic) approaches may arise; in particular, the latter account for subthreshold excitations, for example, $\rho+N \rightarrow N^{*}(1520)$, which induce additional broadening and associated enhancement of the low-mass part in the $\rho$ spectral function (also causing marked deviations from a Breit-Wigner shape). Appreciable mass shifts are typically found in mean-field approximations (due to large in-medium scalar fields) or in calculations where the bare parameters of the underlying Lagrangian are allowed to be temperature dependent [22].

An example for dilepton rates following from a $\rho$ spectral function calculated in hot and dense hadronic matter at SPS energies is shown in Figure 2(a). The EM spectral function follows from the $\rho$-meson using VDM, (3), although corrections to VDM are necessary for quantitative descriptions of the EM couplings in the baryon sector $[23,24]$. When extrapolated to temperatures around $T_{\mathrm{pc}}$, the resonance peak has essentially vanished leading to a structureless emission rate with a large enhancement in the mass region below the free $\rho$ mass. The decomposition of the rate into in-medium selfenergy contributions illustrates the important role of the pion cloud modifications and of multiple low-energy excitations below the free $\rho$ mass, for example, resonance-hole $B N^{-1}$, that is, $\rho+N \rightarrow B$ for off-shell $\rho$-mesons. The hadronic medium effects are slightly reduced at collider energies (Figure 2(b)), where a faint resonance structure appears to survive at around $T_{\mathrm{pc}}$ (it is significantly more suppressed at $T=180 \mathrm{MeV}$ ). A recent calculation in a similar framework, combing thermal field theory with effective hadron Lagrangians [25] and including both finite-temperature and -density contributions to the $\rho$ self-energy through baryon and meson resonances, shows fair agreement with the results shown in Figure 2(a).

2.2. Quark-Gluon Plasma. In a perturbative QGP (pQGP), the leading-order ( $\mathrm{LO})$ mechanism of dilepton production is EM quark-antiquark annihilation as following from a free quark current in (2). The corresponding EM spectral function is essentially given by the "naive quark model" curve in Figure 1, extended all the way down to vanishing mass,

$$
\begin{aligned}
\operatorname{Im} \Pi_{\mathrm{EM}}^{\mathrm{pQGP}} & =-\frac{C_{\mathrm{EM}} N_{c}}{12 \pi} M^{2}\left(1+\frac{2 T}{q} \ln \left[\frac{1+x_{+}}{1+x_{-}}\right]\right) \\
& \equiv \frac{C_{\mathrm{EM}} N_{c}}{12 \pi} M^{2} \widehat{f}_{2}\left(q_{0}, q ; T\right),
\end{aligned}
$$

where $C_{\mathrm{EM}} \equiv \sum_{q=u, d, s} e_{q}^{2}$ (an additional phase-space factor occurs for finite current quark masses $)$ and $x_{ \pm}=\exp \left[-\left(q_{0} \pm\right.\right.$ q)/2T]. Finite-temperature corrections are induced by a quantum-statistical Pauli-blocking factor (written for $\mu_{q}=0$ ) which produces a nontrivial 3-momentum dependence [26]; for $q=0$, it simplifies to $\widehat{f}_{2}\left(q_{0}, q=0 ; T\right)=\left[1-2 f^{F}\left(q_{0} / 2\right)\right]$, where $f^{F}$ is the thermal Fermi distribution. The pertinent 3momentum integrated dilepton rate is structureless (cf. longdashed curve in Figure 2(b)). It's finite value at $M=0$ implies that no real photons can be produced from this mechanism.

A consistent implementation of $\alpha_{s}$ corrections in a thermal QGP at vanishing quark chemical potential has been achieved by resumming the hard-thermal-loop (HTL) action [27]. Quarks and gluons acquire thermal masses $m_{q, g}^{\text {th }} \sim g T$, but bremsstrahlung-type contributions lead to a marked enhancement of the rate over the LO pQCD results (cf. the dash-dotted line in Figure 2(b)).

Recent progress in calculating dilepton rates nonperturbatively using thermal lattice QCD (IQCD) has been reported in $[14,15,28]$. The basic quantity computed in these simulations is the Euclidean-time correlation function which is related to the spectral function, $\rho_{V} \equiv-2 \operatorname{Im} \Pi_{i}^{i}$, via

$$
\Pi_{V}(\tau, q ; T)=\int_{0}^{\infty} \frac{d q_{0}}{2 \pi} \rho_{V}\left(q_{0}, q ; T\right) \frac{\cosh \left[q_{0}(\tau-1 / 2 T)\right]}{\sinh \left[q_{0} / 2 T\right]} .
$$



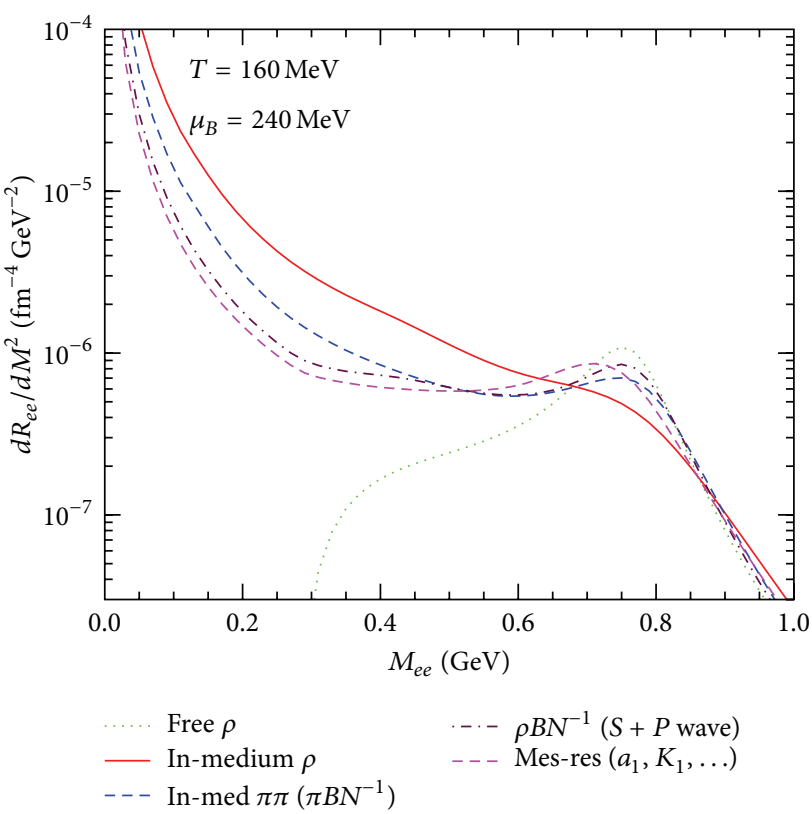

(a)

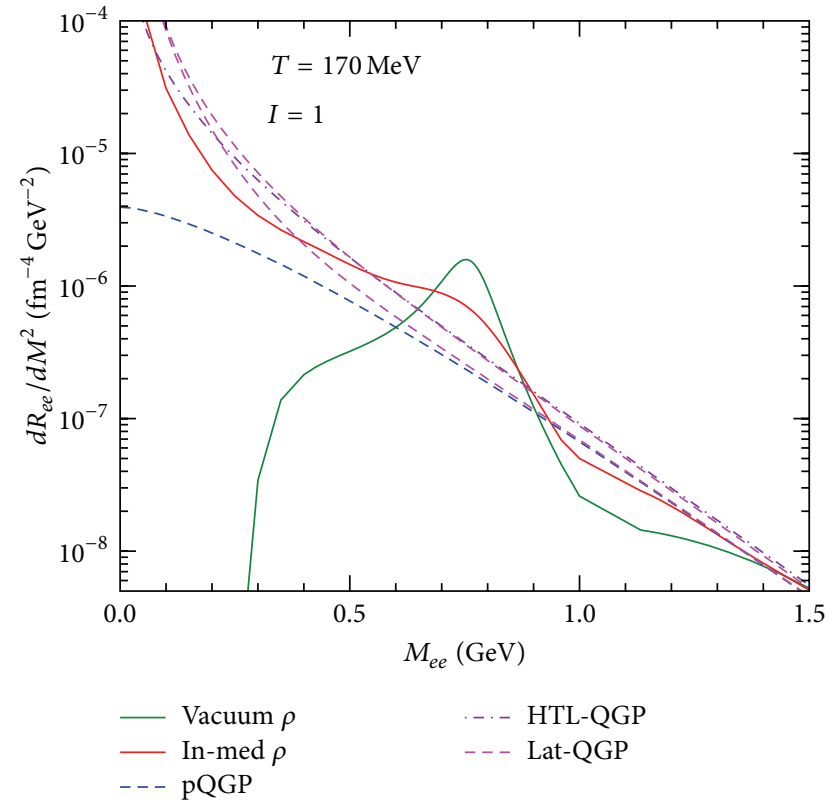

(b)

FIGURE 2: Dilepton rates from hot QCD matter in the isovector $(\rho)$ channel. (a) Effective hadronic Lagrangian plus many-body approach for the in-medium $\rho$ spectral function (solid line) at a temperature and chemical potential characteristic for chemical freezeout at full SPS energy; the effects of in-medium pion-cloud (long-dashed line), baryon resonances (dash-dotted line), and meson resonances (short-dashed line) are shown separately along with the rate based on the vacuum spectral function (dotted line). (b) Comparison of free and in-medium hadronic and partonic calculations at temperature $T=170 \mathrm{MeV}$ and small baryon chemical potential characteristic for RHIC and LHC conditions; the free and in-medium hadronic rates are based on [35, 36]; the "lat-QGP" rates (2 short-dashed lines) are based on fits to the $q=01 \mathrm{QCD}$ rate with extensions to finite 3-momentum utilizing perturbative photon rates (see Section 2.2 for details).

Results for $\Pi_{V}$ obtained in quenched QCD for $T=1.45 T_{c}$ at vanishing $q$ (in which case $M=q_{0}$ ) are shown by the data points in Figure 3(a), normalized to the free (noninteracting) pQGP limit. At small $\tau$, corresponding to large energies in the spectral function, this ratio tends to one as expected for the perturbative limit. For larger $\tau$, a significant enhancement develops which is associated with a corresponding enhancement in the low-energy (or low-mass) regime of the spectral function (and thus dilepton rate). This enhancement may be quantified by making an ansatz for the spectral function in terms of a low-energy Breit-Wigner part plus a perturbative continuum [14],

$$
\rho_{V}^{i i}\left(q_{0}\right)=S_{\mathrm{BW}} \frac{q_{0} \Gamma / 2}{q_{0}^{2}+\Gamma^{2} / 4}+\frac{C_{\mathrm{EM}} N_{c}}{2 \pi}(1+\kappa) q_{0}^{2} \tanh \left(\frac{q_{0}}{4 T}\right)
$$

(note that $\left.\tanh \left(q_{0} / 4 T\right)=1-2 f^{F}\left(q_{0} / 2\right)\right)$. The strength $\left(S_{\mathrm{BW}}\right)$ and width $(\Gamma)$ of the Breit-Wigner, as well as a perturbative $\alpha_{s}$ correction $(\kappa)$, are then fit to the Euclidean correlator. The large- $\tau$ enhancement in the correlator generates an appreciable low-energy enhancement in the spectral function (cf. Figure 3(b)). The zero-energy limit of the spectral function defines a transport coefficient, the electric conductivity, $\sigma_{\mathrm{EM}}=(1 / 6) \lim _{q_{0} \rightarrow 0}\left(\rho_{V}^{i i} / q_{0}\right)$. Similar to the viscosity or heavy-quark diffusion coefficient, a small value for $\sigma_{\mathrm{EM}}$, implied by a large value for $\Gamma$, indicates a strong coupling of the medium; for example, in pQCD, $\sigma_{\mathrm{EM}} \propto T / \alpha_{s}^{2}[29]$.
The results for the dilepton rate (or spectral function) at a smaller temperature of $1.1 T_{c}$ are found to be similar to the ones at $1.45 T_{c}$ [28], suggesting a weak temperature dependence in this regime. Note, however, that the phase transition in quenched QCD is of first order; that is, a stronger variation is expected when going across $T_{c}$. Recent results for twoflavor QCD [15] also indicate rather structureless spectral functions similar to the quenched results. Ultimately, at sufficiently small temperatures, the lattice computations should recover a $\rho$-meson resonance peak; it will be interesting to see at which temperatures this occurs.

For practical applications, a finite 3-momentum dependence of the lQCD dilepton rate is needed, which is currently not available from the simulations. We here propose a "minimal" construction which is based on a matching to the 3momentum dependence obtained from the LO pQCD photon rate [30]. The latter reads

$$
\begin{aligned}
q_{0} \frac{d R_{\gamma}}{d^{3} q} & =-\frac{\alpha_{\mathrm{EM}}}{\pi^{2}} \operatorname{Im} \Pi_{T}(M=0, q) f^{B}\left(q_{0}, T\right) \\
& =\frac{C_{\mathrm{EM}} \alpha \alpha_{S}}{2 \pi^{2}} T^{2} f^{B}\left(q_{0}, T\right) \ln \left(1+\frac{2.912}{4 \pi \alpha_{s}} \frac{q_{0}}{T}\right) .
\end{aligned}
$$

The idea is now to adopt the transverse part of the EM spectral function as given by (7) for the 3-momentum dependence of 


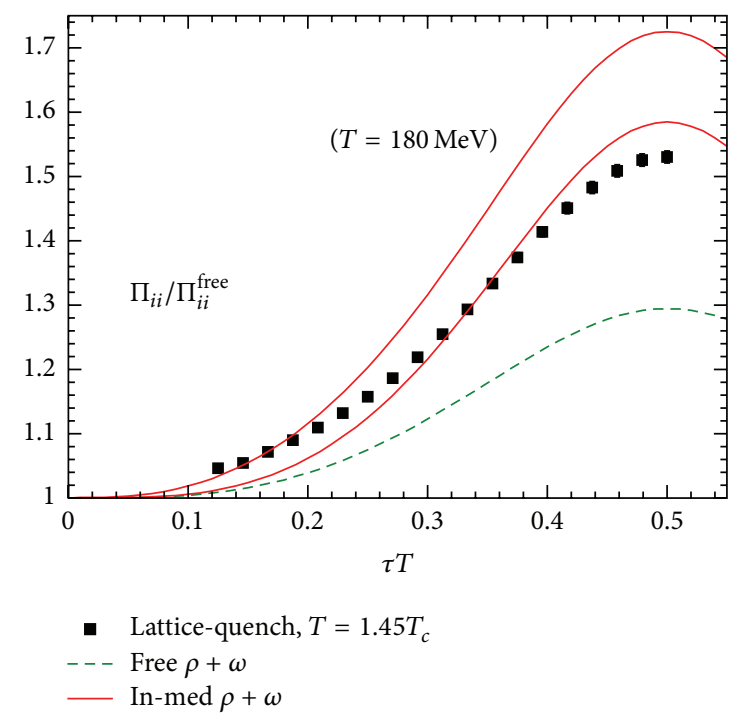

(a)

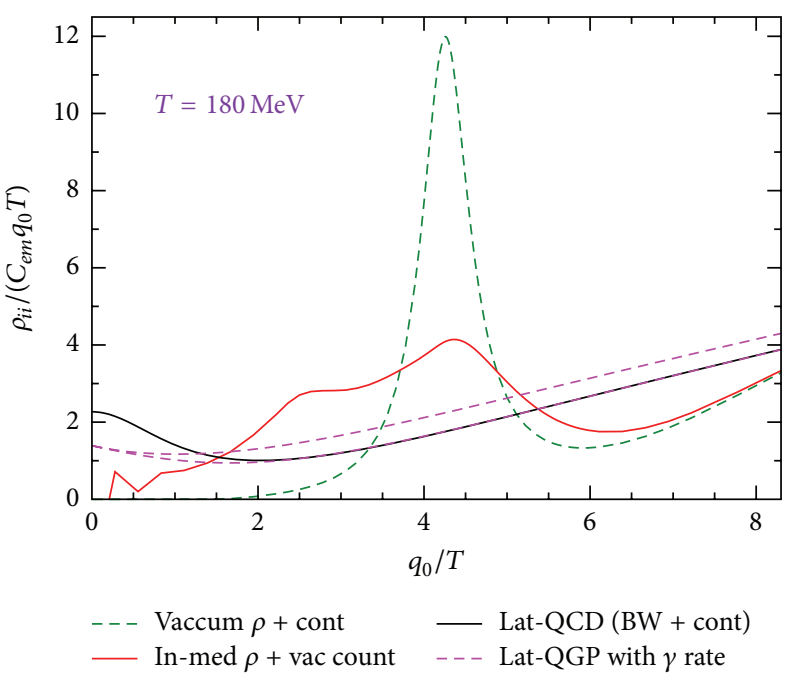

(b)

FIGURE 3: (a) Euclidean correlators of the EM current as computed in quenched thermal lQCD (data points) [14], compared to results from integrating hadronic spectral functions using (5) without (dashed green line) and with in-medium effects (red lines, with free and in-medium continuum threshold) [37]. (b) Vector-isovector spectral functions at $q=0$ corresponding to the Euclidean correlators in (a) in vacuum (green dashed line), in hadronic matter calculated from many-body theory at $T=180 \mathrm{MeV}$ [13] (red solid line), and in a gluon plasma at $1.4 T_{c}$ extracted from thermal lattice-QCD (black solid line) [14]; the 3-momentum extended lQCD rates according to (8) are shown for $K=2$ (short-dashed lines, with (lower) and without (upper) form factor correction).

the spectral function in (6) by replacing the Breit-Wigner part with it; that is,

$$
\begin{aligned}
-\operatorname{Im} \Pi_{T}= & \frac{C_{\mathrm{EM}} N_{c}}{12 \pi} M^{2} \\
& \times\left(\widehat{f}_{2}\left(q_{0}, q ; T\right)\right. \\
& \left.\quad+2 \pi \alpha_{s} \frac{T^{2}}{M^{2}} K F\left(M^{2}\right) \ln \left(1+\frac{2.912}{4 \pi \alpha_{s}} \frac{q_{0}}{T}\right)\right) \\
\equiv & \frac{C_{\mathrm{EM}} N_{c}}{12 \pi} M^{2}\left(\widehat{f}_{2}\left(q_{0}, q ; T\right)+Q_{\mathrm{LAT}}^{T}(M, q)\right) .
\end{aligned}
$$

Here, we have introduced a $K$ factor into $Q_{\mathrm{LAT}}^{T}$, which serves two purposes: (i) with $K=2$, it rather accurately accounts for the enhancement of the complete LO photon rate calculation [31] over the rate in (7); (ii) it better reproduces the lowenergy regime of the lQCD spectral function; for example, for $K=2$, the electric conductivity following from (8) is $\sigma_{\mathrm{EM}} / T \simeq$ $0.23 C_{\mathrm{EM}}$, not far from the lQCD estimate with the fit ansatz (6), $\sigma_{\mathrm{EM}} / T \simeq(0.37 \pm 0.01) C_{\mathrm{EM}}$ (also compatible with [32]; the systematic uncertainty in the lattice result, due to variations in the ansatz, is significantly larger). The resulting spectral function (upper dashed line in Figure 3(b)) somewhat overestimates the IQCD result at high energies, where the latter coincides with the annihilation term. This can be improved by an additional form factor, $F\left(M^{2}\right)=\Lambda^{2} /\left(\Lambda^{2}+M^{2}\right)$, resulting in the lower dashed line in Figure 3(b) (using $\Lambda=2 T$ ).
Finally, care has to be taken to include a finite longitudinal part which develops in the timelike regime. Here, we employ a dependence that follows, for example, from standard constructions of gauge-invariant $S$-wave $\rho$-baryon interactions, yielding $\Pi_{L}=\left(M^{2} / q_{0}^{2}\right) \Pi_{T}$ [34]. Thus, we finally have

$$
Q_{\mathrm{LAT}}^{\mathrm{tot}}=\frac{1}{3}\left(2 Q_{\mathrm{LAT}}^{T}+Q_{\mathrm{LAT}}^{L}\right)=\frac{1}{3} Q_{\mathrm{LAT}}^{T}\left(2+\frac{M^{2}}{q_{0}^{2}}\right) \text {. }
$$

The IQCD results for the isovector spectral function are compared to hadronic calculations in Figure 3(b). Close to the phase transition temperature, the "melting" of the inmedium $\rho$ spectral function suggests a smooth transition from its prominent resonance peak in vacuum to the rather structureless shape extracted from $1 Q C D$, signaling a transition from hadronic to partonic degrees of freedom. It would clearly be of interest to extract the conductivity from the hadronic calulations, which currently is not well resolved from the $q=0, q_{0} \rightarrow 0$ limit of the spectral function. The mutual approach of the nonperturbative hadronic and IQCD spectral functions is also exhibited in the 3-momentum integrated dilepton rate shown in Figure 2(b), especially when compared to the different shapes of the LO pQCD and vacuum hadronic rates. Arguably, the in-medium hadronic rate still shows an indication of a broad resonance. A smooth matching of the rates from above and below $T_{\mathrm{pc}}$ might therefore require some additional medium effects in the hot and dense hadronic medium and/or the emergence of resonance correlations in the $q \bar{q}$ correlator in the QGP. Unless otherwise noted, the thermal emission rates used in the calculations of dilepton spectra discussed below will be based 


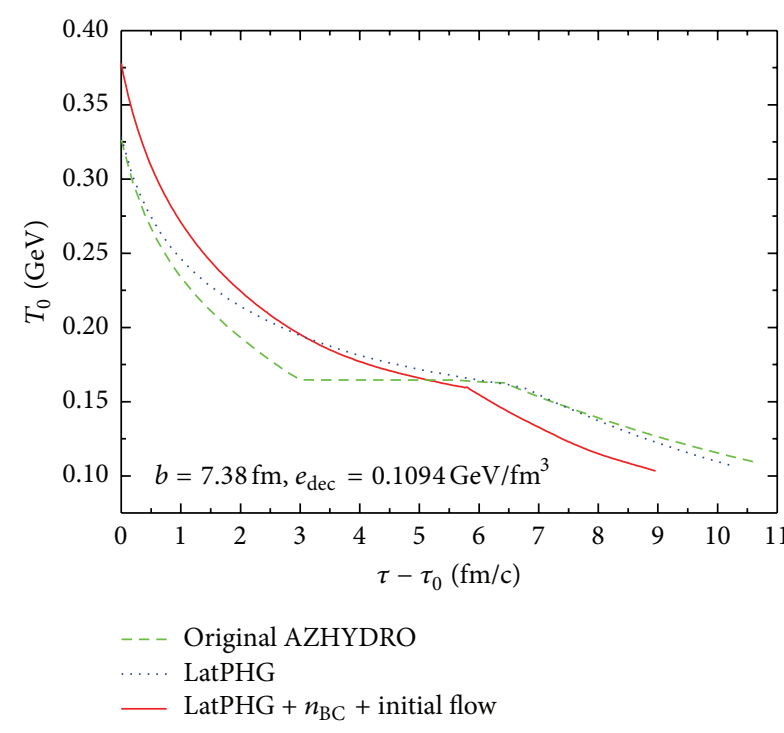

(a)

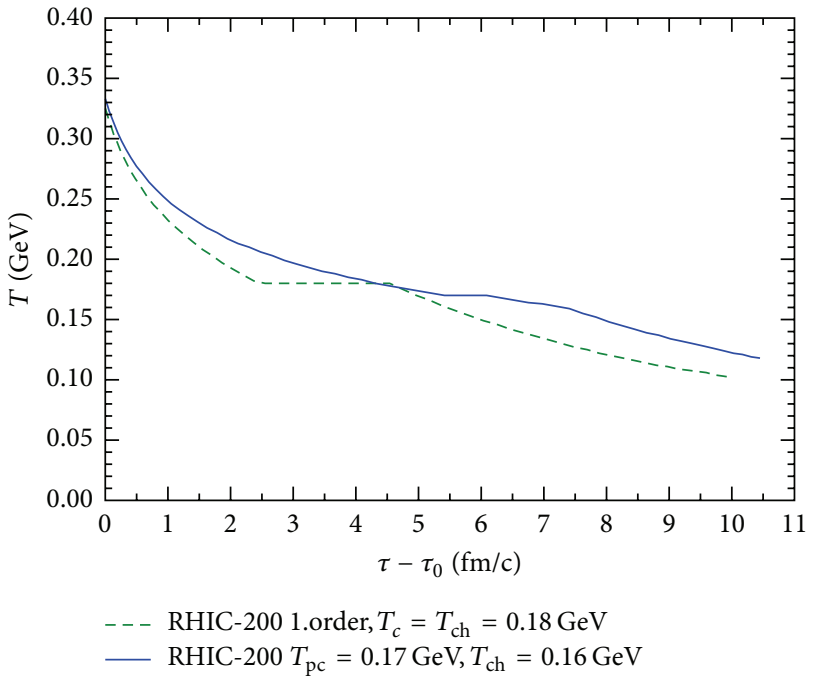

(b)

FIGURE 4: Time evolution of fireball temperature in semicentral $\mathrm{Au}-\mathrm{Au}(\sqrt{s}=0.2 \mathrm{GeV})$ collisions at RHIC within (the central cell of) ideal hydrodynamics (a) [42] and an expanding fireball model (b). The dashed green and dotted blue lines in (a) are to be compared to the dashed green and solid blue lines in (b), respectively.

on the in-medium hadronic rates of [35] and the lQCDinspired QGP rates [14], extended to finite 3-momentum as constructed above (with $K=2$ and form factor).

\section{Dilepton Spectra at RHIC and LHC}

The calculation of dilepton mass and transverse-momentum $\left(q_{t}\right)$ spectra, suitable for comparison to data in heavyion collisions, requires an integration of the thermal rates of hadronic matter and QGP over a realistic space-time evolution of the AA reaction:

$$
\frac{d N_{l l}}{d M}=\int d^{4} x \frac{M d^{3} q}{q_{0}} \frac{d N_{l l}}{d^{4} x d^{4} q} .
$$

In addition to the thermal yield, nonthermal sources have to be considered, for example, primordial Drell-Yan annihilation and electromagnetic final-state decays of long-lived hadrons. We will briefly discuss space-time evolutions in Section 3.1 and nonthermal sources in Section 3.2, before proceeding to a more detailed discussion of thermal spectra and comparisons to data, as available, in Sections 3.3, 3.4, and 3.5 for full RHIC energy, the beam-energy scan, and LHC, respectively.

3.1. Medium Expansion. The natural framework to carry out the space-time integral over the dilepton rate in URHICs is relativistic hydrodynamics. The application of this approach to AA collisions at RHIC and LHC works well to describe bulk hadron observables (e.g., $p_{t}$ spectra and elliptic flow) up to momenta of $p_{t} \simeq 2-3 \mathrm{GeV}$, which typically comprises more than $90 \%$ of the total yields. Some uncertainties remain, for example, as to the precise initial conditions at thermalization, viscous corrections, or the treatment of the late stages where the medium becomes dilute and the hadrons decouple (see, e.g., [38] for a recent review). Another key ingredient is the equation of state $(\mathrm{EoS})$ of the medium, $\varepsilon(P)$, which drives its collective expansion. Figure 4(a) illustrates the effects of updating a previously employed bag-model EoS (a quasiparticle QGP connected to a hadron resonance gas via a firstorder phase transition) [39] by a recent parametrization of a nonperturbative QGP EoS from lQCD data [40, 41] (continuously matched to a hadron-resonance gas at $T_{\mathrm{pc}}=170 \mathrm{MeV}$ ) [42]; within a 2+1-D ideal hydro calculation, the most notable change is a significant increase of the temperature (at fixed entropy density) in the regime just above the transition temperature (up to ca. $30 \mathrm{MeV}$ at the formerly onset of the firstorder transition). Together with the fact that the hadronic portion of the formerly mixed phase is now entirely associated with the QGP, this will lead to an increase (decrease) of the QGP (hadronic) contribution to EM radiation relative to the first-order scenario. In addition, the harder lattice EoS induces a stronger expansion leading to a slightly faster cooling and thus reduction in the lifetime by about $5 \%$. This effect becomes more pronounced when modifying the initial conditions of the hydrodynamic evolution, for example, by introducing a more compact spatial profile (creating larger gradients) and/or initial transverse flow (associated with interactions prior to the thermalization time, $\tau_{0}$ ) [42] (cf. the solid line in Figure 4(a)). The resulting more violent expansion plays an important role in understanding the HBT radii of the system [43]. The relevance for EM radiation pertains to reducing the fireball lifetime by up to $\sim 20 \%$.

More simplistic parametrizations of the space-time evolution of AA collisions have been attempted with longitudinally and transversely expanding fireballs. With an appropriate choice of the transverse acceleration (in all applications below 
it is taken as $a_{t}=0.12 / \mathrm{fm}$ at the surface), an approximate reproduction of the basic features (timescales and radial flow) of hydrodynamic evolutions can be achieved, see Figure 4(b). Most of the results shown in the remainder of this article are based on such simplified fireball parametrizations, utilizing the EoS of [42] where a parametrization of IQCD results is matched with a hadron resonance gas at $T_{\mathrm{pc}}=170 \mathrm{MeV}$ and subsequent chemical freezeout at $T_{\mathrm{ch}}=160 \mathrm{MeV}$ (see also [44]). We note that the use of this EoS, together with the lQCD-based QGP emission rates, constitutes an update of our earlier calculations [45] where a quasiparticle bag-model EoS was employed in connection with HTL rates in the QGP. We have checked that the previous level of agreement with the acceptance-corrected NA60 spectra is maintained, which is essentially due to the duality of the QGP and hadronic rates around $T_{\mathrm{pc}}$ (a more detailed account in the context of the SPS dilepton data will be given elsewhere [46]). For our discussion of collider energies below, the initialization (or thermalization times) are chosen at $0.33 \mathrm{fm} / c$ at full RHIC energy (increasing smoothly to $1 \mathrm{fm} / c$ at $\sqrt{s}=20 \mathrm{GeV}$ ) and $0.2 \mathrm{fm} / c$ in the LHC regime. This results in initial temperatures of $225 \mathrm{MeV}$ and $330 \mathrm{MeV}$ in minimum-bias (MB) Au$\mathrm{Au}$ collisions at 20 and $200 \mathrm{GeV}$, respectively, increasing to $\sim 380 \mathrm{MeV}$ in central Au-Au(200 GeV) and $\sim 560(620) \mathrm{MeV}$ in central $\mathrm{Pb}-\mathrm{Pb}$ at 2.76(5.5) ATeV. These values differ slightly from previous calculations with a quasiparticle EoS; they are also sensitive to the initial spatial profile (cf. Figure 4(a)). However, for our main objective of calculating low-mass dilepton spectra, the initial temperature has little impact.

3.2. Nonthermal Sources. In addition to thermal radiation from the locally equilibrated medium, dilepton emission in URHICs can arise from interactions prior to thermalization (e.g., Drell-Yan annihilation) and from EM decays of longlived hadrons after the fireball has decoupled (e.g., Dalitz decays $\pi^{0}, \eta \rightarrow \gamma l^{+} l^{-}$or $\omega, \phi \rightarrow l^{+} l^{-}$). Furthermore, paralleling the structure in hadronic spectra, a nonthermal component from hard production will feed into dilepton spectra, for example, via bremsstrahlung from hard partons traversing the medium [47] or decays of both short- and longlived hadrons which have not thermalized with the bulk (e.g., "hard" $\rho$-mesons or long-lived EM final-state decays). Hadronic final-state decays (including the double semileptonic decay of two heavy-flavor hadrons originating from a $c \bar{c}$ or $b \bar{b}$ pair produced together in the same hard process) are commonly referred to as the "cocktail," which is routinely evaluated by the experimental collaborations using the vacuum properties of each hadron with $p_{t}$ spectra based on measured spectra or appropriately extrapolated using thermal blast-wave models. In URHICs, the notion of the cocktail becomes problematic for short-lived resonances whose lifetime is comparable to the duration of the freezeout process of the fireball (e.g., for $\rho, \Delta$, etc.). In their case, a better approximation is presumably to run the fireball an additional $\sim 1 \mathrm{fm} / c$ to treat their final-decay contribution as thermal radiation including medium effects. However, care has to be taken in evaluating their dilepton $p_{t}$-spectra, as the latter are slightly different for thermal radiation and final-state decays (cf. [45] for a discussion and implementation of this point). For light hadrons at low $p_{t}$, the cocktail scales with the total number of charged particles, $N_{\mathrm{ch}}$, at given collision energy and centrality, while for hard processes, a collision-number scaling $\propto N_{\text {coll }}$ is in order (and compatible with experiment where measured, modulo the effects of "jet quenching"). The notion of "excess dileptons" is defined as any additional radiation observed over the cocktail, sometimes quantified as an "enhancement factor" in a certain invariant-mass range. The excess radiation is then most naturally associated with thermal radiation, given the usual limitation where hard processes take over, that is, $M, q_{t} \leqslant 2-3 \mathrm{GeV}$.

3.3. RHIC-200. We start our discussion of low-mass dilepton spectra at full RHIC energy where most of the current experimental information at collider energies is available, from both PHENIX [48] and STAR [49] measurements.

3.3.1. Invariant-Mass Spectra. Figure 5 shows the comparison of thermal fireball calculations with low-mass spectra from STAR [49]. As compared to earlier calculations with a bagmodel EoS [13], the use of IQCD-EoS and emission rates for the QGP enhances the pertinent yield significantly. It is now comparable to the in-medium hadronic contribution for masses below $M \simeq 0.3 \mathrm{GeV}$ and takes over in the intermediate-mass region $(M \gtrsim 1.1 \mathrm{GeV})$. The hadronic part of the thermal yield remains prevalent in a wide range around the free $\rho$ mass, with a broad resonance structure and appreciable contributions from $4 \pi$ annihilation for $M \gtrsim 0.9 \mathrm{GeV}$. Upon adding the thermal yield to the final-state decay cocktail by the STAR collaboration (without $\rho$ decay), the MB data are well described. For the central data, a slight overestimate around $M \simeq 0.2 \mathrm{GeV}$ and around the $\omega$ peak is found. A similar description [51] of the STAR data arises in a viscous hydrodynamic description of the medium using the $\rho$ spectral function from on-shell scattering amplitudes [21] (see also [52]) and in the parton-hadron string dynamics transport approach using a schematic temperature- and densitydependent broadening in a Breit-Wigner approximation of the $\rho$ spectral function [53]. More studies are needed to discern the sensitivity of the data to the in-medium spectral shape, as the latter significantly varies in the different approaches. For the PHENIX data (not shown), the enhancement as recently reported in [54] for noncentral collisions (carried out with the hadron-blind detector (HBD) upgrade) agrees with earlier measurements [48], is consistent with the STAR data, and thus should also agree with theory. For the most central Au-Au data, however, a large enhancement was reported in [48], which is well above theoretical calculations with broad spectral functions $[13,53,55,56]$, even in the MB sample. More "exotic" explanations of this effect, which did not figure at the SPS, for example, a Bose-condensed like glasma in the pre-equilibrium stages [57], have been put forward to explain the "PHENIX puzzle." However, it is essential to first resolve the discrepancy on the experimental side, which is anticipated with the HBD measurement for central collisions.

To quantify the centrality dependence of the thermal radiation (or excess) yield, one commonly introduces an 

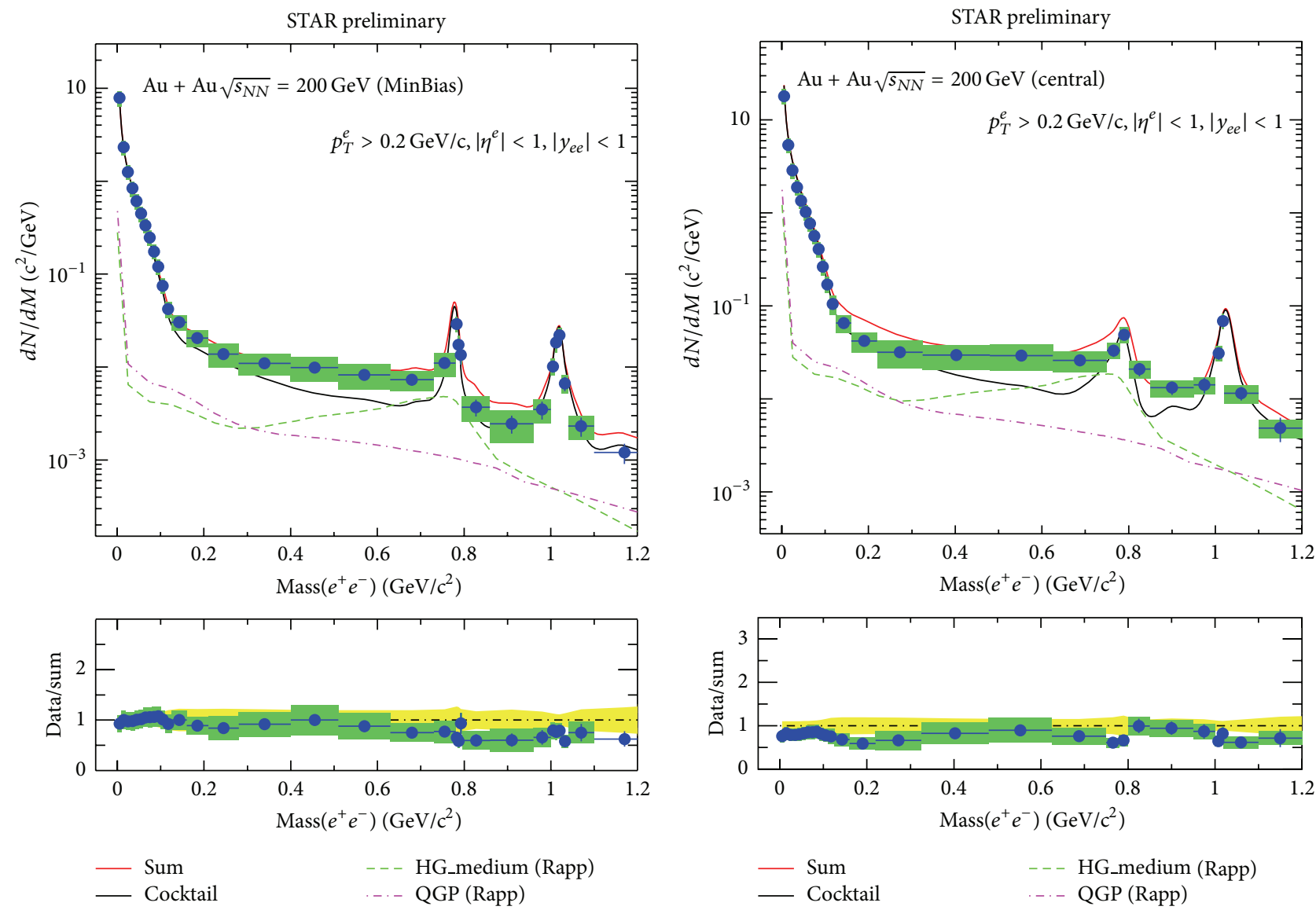

(a)

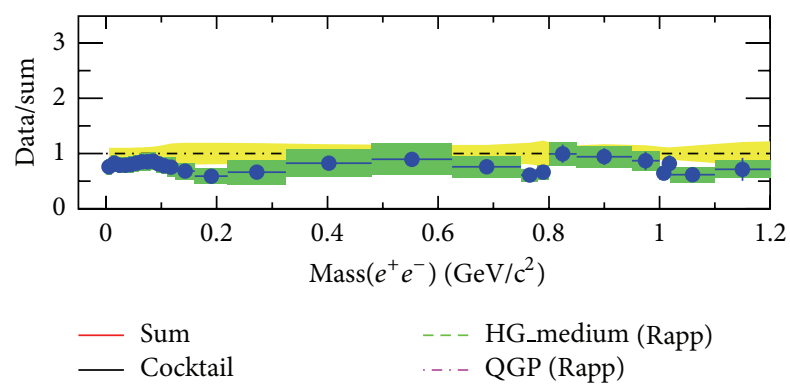

(b)

Figure 5: Dilepton invariant-mass spectra in $\mathrm{Au}-\mathrm{Au}(200 \mathrm{AGeV})$ for minimum-bias (a) and central (b) collisions. Theoretical calculations for thermal radiation from a nonperturbative QGP and in-medium hadronic spectral functions are compared to STAR data [49,50].

exponent $\alpha_{c}$ as $Y_{M}\left(N_{\mathrm{ch}}\right) / N_{\mathrm{ch}}=C N_{\mathrm{ch}}^{\alpha_{c}}$, which describes how the excess (or thermal) yield in a given mass range scales relative to the charged-particle multiplicity. For full RHIC energy, the theoretical calculation gives $\alpha_{c} \simeq 0.45$ (with a ca. $10 \%$ error), similar to what had been found for integrated thermal photon yields [58].

3.3.2. Transverse-Momentum Dependencies. When corrected for acceptance, invariant-mass spectra are unaffected by any blue-shift of the expanding medium, which renders them a pristine probe for in-medium spectral modifications. However, the different collective flow associated with different sources may be helpful in discriminating them by investigating their $q_{t}$ spectra, see, for example, [26, 5964]. As is well known from the observed final-state hadron spectra, particles of larger mass experience a larger blueshift than lighter particles due to collective motion with the expanding medium. Schematically, this can be represented by an effective slope parameter which for sufficiently large masses takes an approximate form of $T_{\text {eff }}=T+M \bar{\beta}^{2}$, where $T$ and $\bar{\beta}$ denote the local temperature and average expansion velocity of the emitting source cell. Dileptons are well suited to systematically scan the mass dependence of $T_{\text {eff }}$ by studying $q_{t}$ spectra for different mass bins (provided the data have sufficient statistics). At the SPS, this has been done by the NA60 collaboration [65], who found a gradual increase in the slope from the dimuon threshold to the $\rho$ mass characteristic for a source of hadronic origin (a.k.a inmedium $\rho$ mesons), a maximum around the $\rho$ mass (late $\rho$ decays), followed by a decrease and leveling off in the intermediate-mass region (IMR, $M \geq 1 \mathrm{GeV}$ ) indicative for early emission at temperatures $T \simeq 170-200 \mathrm{MeV}$ (where at the SPS the collective flow is still small).

Figure 6 shows the $q_{t}$ spectra for thermal radiation from hadronic matter and QGP in MB Au-Au(200 AGeV) in two typical mass regions where either of the two sources dominates. In the low-mass region (LMR), both sources have a surprisingly similar slope $\left(T_{\text {slope }} \simeq 280-285 \mathrm{MeV}\right)$, reiterating that the emission is from mostly around $T_{\mathrm{pc}}$ where the slope of both sources is comparable (also recall from Figure 5 that in the mass window $M=0.3-0.7 \mathrm{GeV}$ the QGP emission is largest at the lower mass end, while the hadronic one is more weighted toward the higher end). For definiteness, assuming $T=170 \mathrm{MeV}$ and $M=0.5 \mathrm{GeV}$, one finds that $\bar{\beta} \simeq 0.45-0.5$, which is right in the expected range [42]. On the other hand, in the IMR, where the QGP dominates, the hadronic 


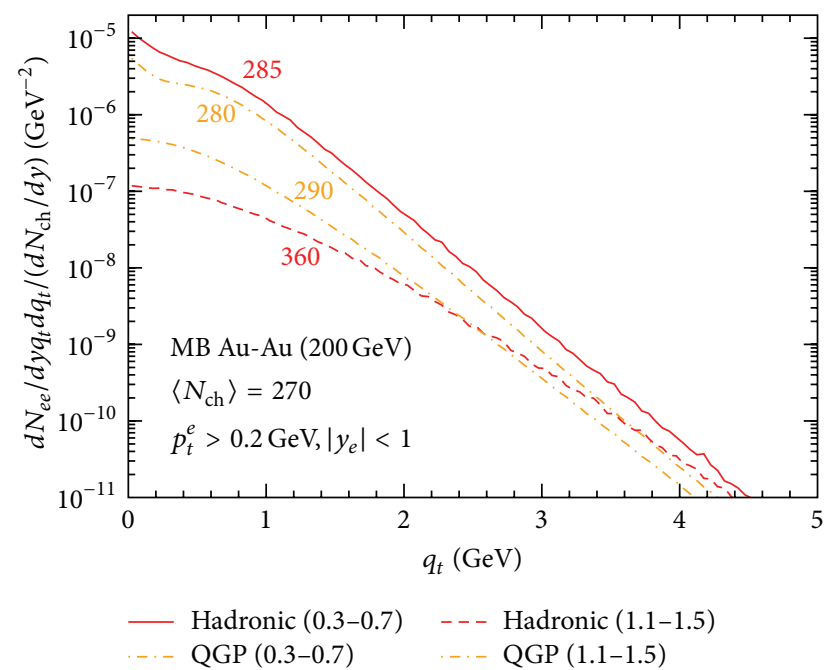

Figure 6: Dilepton transverse-momentum spectra from thermal radiation of QGP and hadronic matter in $\mathrm{MB} \mathrm{Au}-\mathrm{Au}(200 \mathrm{AGeV})$ collisions. The numbers next to each curve indicate the effective slope parameter, $T_{\text {eff }}(\mathrm{MeV})$, as extracted from a two parameter fit using $d N /\left(q_{t} d q_{t}\right)=C \exp \left[-M_{t} / T_{\text {eff }}\right]$ [45] with the transverse mass $M_{t}=\sqrt{M^{2}+q_{t}^{2}}$ and an average mass of $0.5 \mathrm{GeV}$ and $1.25 \mathrm{GeV}$ for the low- and intermediate-mass windows, respectively.

slope has significantly increased to ca. $360 \mathrm{MeV}$ due to the larger mass in the collective-flow term. On the other hand, the slope of the QGP emission has only slightly increased over the LMR, indicating that the increase in mass in the flow term is essentially offset by an earlier emission temperature, as expected for higher mass (for hadronic emission, the temperature is obviously limited by $T_{\mathrm{pc}}$ ). Consequently, at RHIC, the effective slope of the total thermal radiation in the IMR exceeds the one in the LMR, contrary to what has been observed at SPS. Together with blue-shift free temperature measurements from slopes in invariant-mass spectra, this provides a powerful tool for disentangling collective and thermal properties through EM radiation from the medium.

Alternatively, one can investigate the mass spectra in different momentum bins, possibly revealing a $q_{t}$-dependence of the spectral shape, as was done for both $e^{+} e^{-}$data in $\mathrm{Pb}-\mathrm{Au}$ [66] and $\mu^{+} \mu^{-}$in In-In [65] at SPS. Calculations for thermal radiation in $\mathrm{Au}-\mathrm{Au}$ at full RHIC energy are shown in Figure 7 for four bins from $q_{t}=0-2 \mathrm{GeV}$. One indeed recognizes that the $\rho$ resonance structure becomes more pronounced as transverse momentum is increased. In the lowest bin, the minimum structure around $M \simeq 0.2 \mathrm{GeV}$ is caused by the experimental acceptance, specifically the single-electron $p_{t}^{e}>$ $0.2 \mathrm{GeV}$, which for vanishing $q_{t}$ suppresses all dilepton yields below $M \simeq 2 p_{t}^{e, \min }=0.4 \mathrm{GeV}$.

3.3.3. Elliptic Flow. Another promising observable to diagnose the collectivity, and thus the origin of the EM emission source, is its elliptic flow $[64,69,70]$. The latter is particularly useful to discriminate early from late(r) thermal emission sources; contrary to the slope parameter, which is subject to an interplay of decreasing temperature and increasing flow, the medium's ellipticity is genuinely small (large) in the early (later) phases. Figure 8(a) shows hydrodynamic calculations of the inclusive thermal dilepton $v_{2}$ as a function of invariant mass (using the same emission rates and EoS as in the previous figures) [67]. One nicely recognizes a broad maximum structure around the $\rho$ mass, indicative for predominantly later emission in the vicinity of its vacuum mass, a characteristic mass dependence (together with an increasing QGP fraction) below, and a transition to a dominant QGP fraction with reduced $v_{2}$ above. All these features are essentially paralleling the mass dependence of the slope parameter at SPS, while the latter exhibits a marked increase at RHIC in the IMR due to the increased radial flow in the QGP and early hadronic phase. Rather similar results are obtained in hydrodynamic calculations with in-medium spectral functions from on-shell scattering amplitudes [51]. When using a less pronounced in-medium broadening, the peak structure in $v_{2}(M)$ tends to become narrower $[64,69,70]$. First measurements of the dilepton- $v_{2}$ have been presented by STAR [68], see Figure 8(b). The shape of the data is not unlike the theoretical calculations, while it is also consistent with the simulated cocktail contribution. Note that the total $v_{2}$ is essentially a weighted sum of cocktail and excess radiation. Thus, if the total $v_{2}$ were to agree with the cocktail, it would imply that the $v_{2}$ of the excess radiation is as large as that of the cocktail. Clearly, future $v_{2}$ measurements with improved accuracy will be a rich source of information.

Significant $v_{2}$ measurements of EM excess radiation have recently been reported in the $M=0$ limit, that is, for direct photons, by both PHENIX [71, 72] and ALICE [73, 74]. A rather large $v_{2}$ signal has been observed in both experiments $[72,74]$, suggestive for rather late emission [75] (see also [7679]). In addition, the effective slope parameters of the excess radiation have been extracted, $T_{\text {eff }}=219 \pm 27 \mathrm{MeV}$ [71] at RHIC-200 and $304 \pm 51 \mathrm{MeV}$ at LHC-2760 [73], which are rather soft once blue-shift effects are accounted for. In fact, these slopes are not inconsistent with the trends in the LMR dileptons when going from RHIC (Figure 6) to LHC (Figure 12). This would corroborate their main origin from around $T_{\mathrm{pc}}$.

3.4. RHIC Beam Energy Scan. A central question for studying QCD phase structure is how the spectral properties of excitations behave as a function of chemical potential and temperature. With the EM (or vector) spectral function being the only one directly accessible via dileptons, systematic measurements as a function of beam energy are mandatory. At fixed target energies, this is being addressed by the current and future HADES efforts $\left(E_{\text {beam }}=1-10 \mathrm{AGeV}\right)[80,81]$, by CBM for $E_{\text {beam }}(\mathrm{Au})$ up to $\sim 35 \mathrm{AGeV}$ [2], and has been measured at SPS energies at $E_{\text {beam }}=158 \mathrm{AGeV}$, as well as in a CERES run at $40 \mathrm{AGeV}$ [82].

At collider energies, a first systematic study of the excitation function of dilepton spectra has been conducted by STAR [68] as part of the beam-energy scan program at RHIC. The low-mass excess radiation develops smoothly when going down from $\sqrt{s_{N N}}=200 \mathrm{GeV}$ via $62 \mathrm{GeV}$ to $20 \mathrm{GeV}$ (cf. Figure 9). Closer inspection reveals that the enhancement factor of excess radiation over cocktail in the region below 


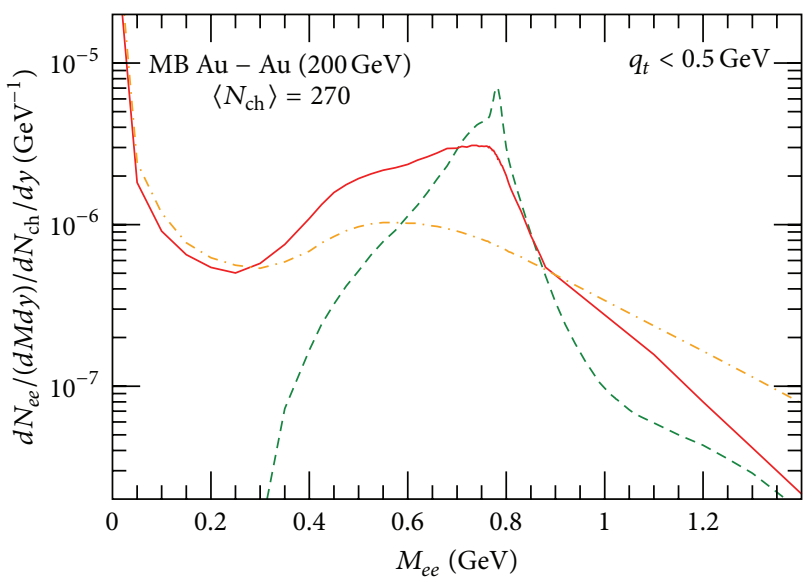

(a)

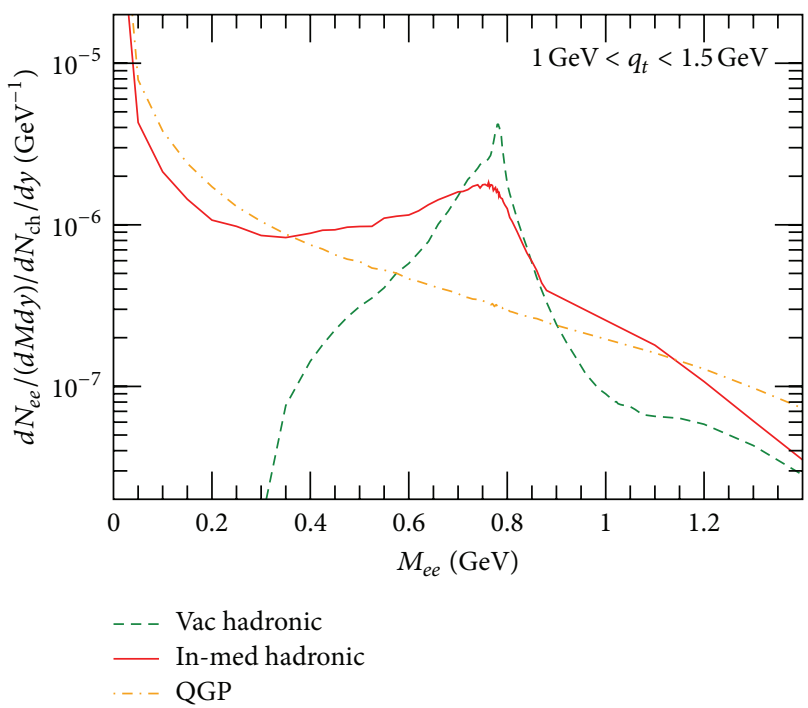

(c)

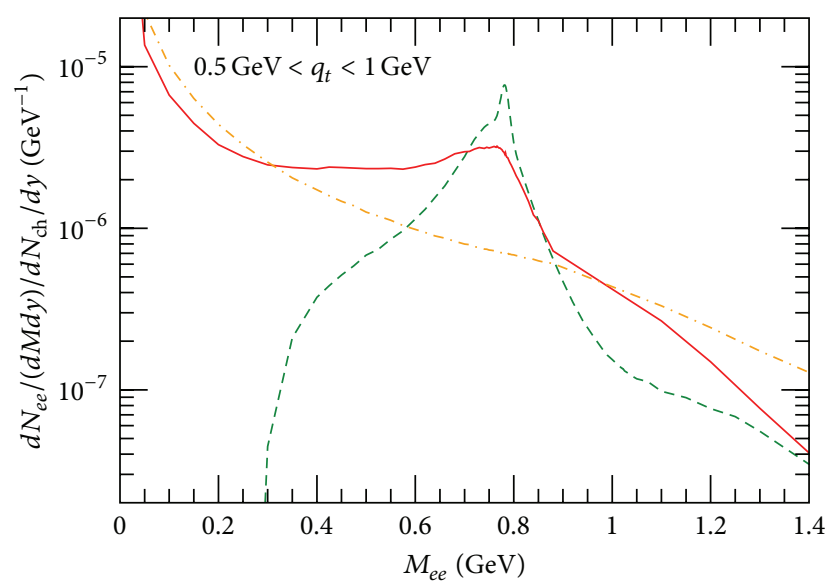

(b)

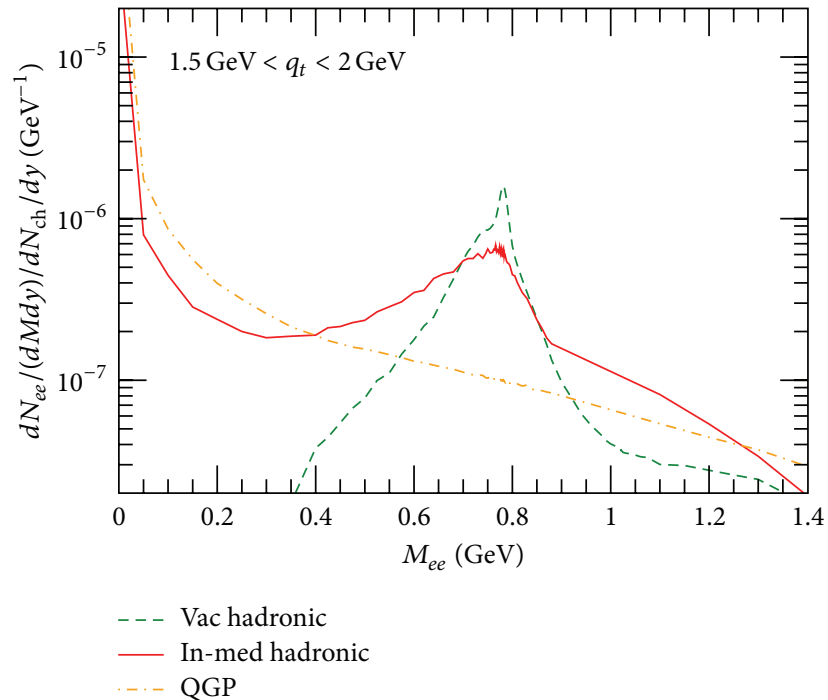

(d)

FIGURE 7: Dilepton invariant mass spectra in different bins of transverse momentum from thermal radiation of QGP (dash-dotted line) and hadronic matter (solid line: in medium, dashed line: vacuum spectral function) in $\mathrm{MB} \mathrm{Au}-\mathrm{Au}(200 \mathrm{AGeV})$ collisions; experimental acceptance as in Figures 5 and 6.

the $\rho$ mass increases as the energy is reduced [68]. An indication of a similar trend was observed when comparing the CERES measurements in $\mathrm{Pb}-\mathrm{Au}$ at $\sqrt{s_{N N}}=17.3 \mathrm{GeV}$ and $8.8 \mathrm{GeV}$. Theoretically, this can be understood by the importance of baryons in the generation of medium effects [24], specifically, the low-mass enhancement in the $\rho$ spectral function. These medium effects become stronger as the beam energy is reduced since the hadronic medium close to $T_{\mathrm{pc}}$ becomes increasingly baryon rich. At the same time, the cocktail contributions, which are mostly made up by meson decays, decrease. The hadronic in-medium effects are expected to play a key role in the dilepton excess even at collider energies. The comparison with the STAR excitation function supports the interpretation of the excess radiation as originating from a melting $\rho$ resonance in the vicinity of $T_{\mathrm{pc}}$.

A major objective of the beam-energy program is the search for a critical point. One of the main effects associated with a second-order endpoint is the critical slowing down of relaxation rates due to the increase in the correlation length in the system. For the medium expansion in URHICs, this may imply an "anomalous" increase in the lifetime of the interacting fireball. If this is so, dileptons may be an ideal tool to detect this phenomenon, since their total yield (as quantified by their enhancement factor) is directly proportional to the duration of emission. The NA60 data have shown that such a lifetime measurement can be carried out with an uncertainty of about $\pm 1 \mathrm{fm} / c$ [45]. In the calculations shown in Figure 9, no critical slowing down has been assumed; as a result, the average lifetime in $\mathrm{MB} \mathrm{Au}-\mathrm{Au}$ collisions increases smoothly from ca. 8 to $10 \mathrm{fm} / c$. Thus, if a critical point were to exist and lead to a, say, $30 \%$ increase in the lifetime in a reasonably localized range of beam energies, dilepton yields ought to be able to detect this signature. This signal would further benefit from the fact that the prevalent radiation arises from around 


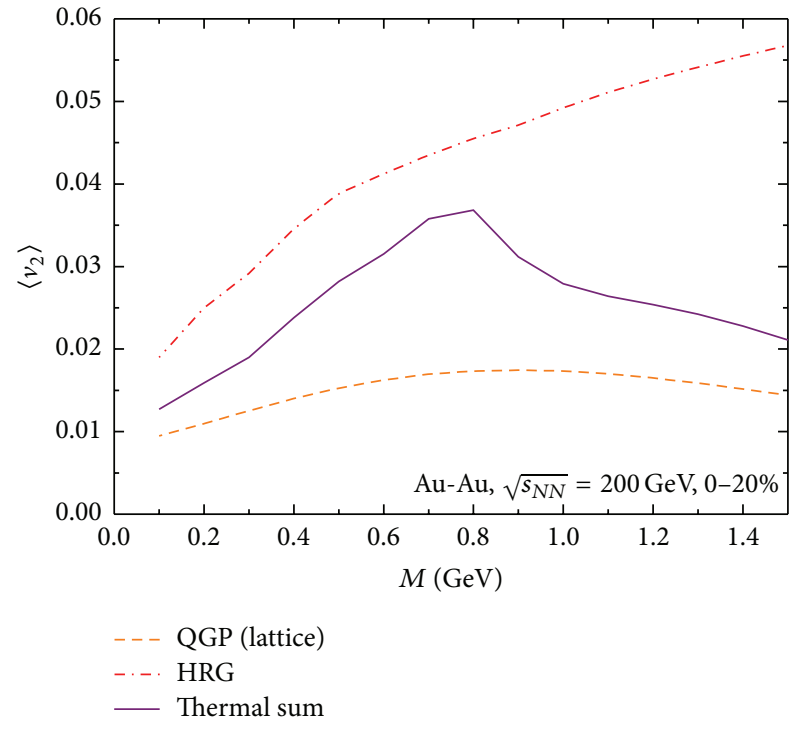

(a)

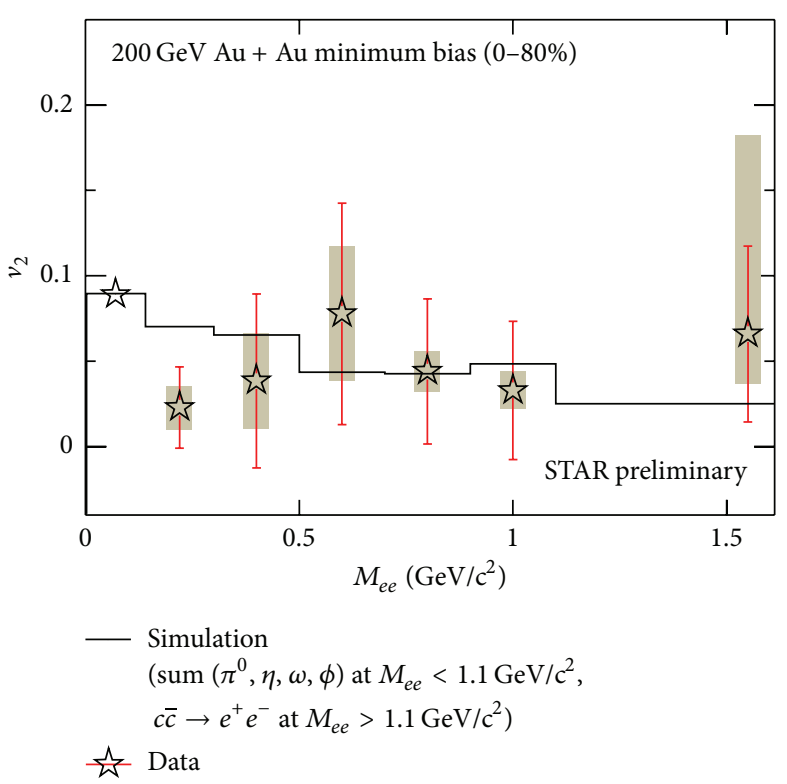

(b)

Figure 8: (a) Inclusive elliptic flow of thermal dileptons in $0-20 \%$ central $\mathrm{Au}-\mathrm{Au}(200 \mathrm{AGeV})$ collisions, calculated within an ideal hydrodynamic model with lattice EoS using 1QCD-based QGP and medium-modified hadronic rates [67]. (b) Dielectron- $v_{2}$ measured by STAR in MB Au-Au [68], including the cocktail contribution; the latter has been simulated by STAR and is shown separately by the solid histogram.

$T_{\mathrm{pc}}$ where the largest effect from the slowing down is expected.

3.5. LHC. The previous section raises the question whether the smooth excitation function of dilepton invariant-mass spectra in the RHIC regime will continue to LHC energies, which increase by another factor of $\sim 20$. On the other hand, the dilepton $q_{t}$ spectra, especially their inverse-slope parameters, indicate an appreciable variation from SPS to RHIC, increasing from ca. 220 to $280 \mathrm{MeV}$ in the LMR, and, more pronounced, from ca. 210 to about $320 \mathrm{MeV}$ in the IMR. This is a direct consequence of the stronger (longer) development of collective flow in the QGP phase of the fireball evolution. This trend will continue at the LHC, as we will see below. In the following Section 3.5.1, we will first discuss the dielectron channel at LHC and highlight the excellent experimental capabilities that are anticipated with a planned major upgrade program of the ALICE detector [83]. In addition, ALICE can measure in the dimuon channel, albeit with somewhat more restrictive cuts whose impact will be illustrated in Section 3.5.2.

3.5.1. Dielectrons. The invariant-mass spectra of thermal radiation at LHC energies show a very similar shape and hadronic/QGP composition as at RHIC energy, see Figure 10. This is not surprising given the virtually identical in-medium hadronic and QGP rates along the thermodynamic trajectories at RHIC and LHC (where $\mu_{B} \ll T$ at chemical freezeout). It implies that the thermal radiation into the LMR is still dominated by temperatures around $T_{\mathrm{pc}}$, with little (if any) sensitivity to the earliest phases. The total yield, on the other hand, increases substantially due to the larger fireball volumes created by the larger multiplicities. More quantitatively, the ( $N_{\mathrm{ch}}$-normalized) enhancement around, for example, $M=0.4 \mathrm{GeV}$, approximately scales as $N_{\mathrm{ch}}^{\alpha_{E}}$ with $\alpha_{E} \simeq 0.8$ relative to central $\mathrm{Au}-\mathrm{Au}$ at full RHIC energy. This is a significantly stronger increase than the centrality dependent enhancement at fixed collision energy, $\alpha_{c} \simeq 0.45$ as quoted in Section 3.3.1.

Detailed simulation studies of a proposed major upgrade of the ALICE detector have been conducted in the context of a pertinent letter of intent [83]. The final results after subtraction of uncorrelated (combinatorial) background are summarized in Figure 11, based on an excess signal given by the thermal contributions in Figure 10. (The thermal yields provided for the simulations were later found to contain a coding error in the author's implementation of the experimental acceptance; the error turns out to be rather inconsequential for the shape and relative composition of the signal, as a close comparison of Figures 10(b) and 11(b) reveals; the absolute differential yields differ by up to $20-30 \%$.) Figure 10(a) shows that the thermal signal is dominant for the most part of the LMR (from ca. $0.2-1 . \mathrm{GeV}$ ), while in the IMR it is outshined by correlated heavy-flavor decays. However, the latter can be effectively dealt with using displaced vertex cuts, while the excellent mass resolution, combined with measured and/or inferred knowledge of the Dalitz spectra of $\pi^{0}$ (from charged pions), $\eta$ (from charged kaons), and $\omega$ (from direct dilepton decays), facilitates a reliable subtraction of the cocktail. The resulting excess spectra shown in Figure 10(b) are of a quality comparable to the NA60 data. This will allow for quantitative studies of the in-medium EM spectral 


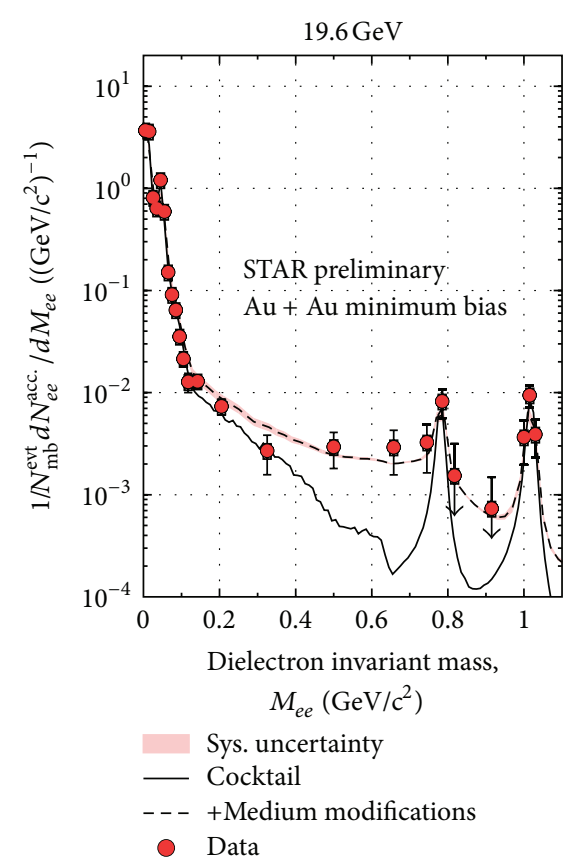

(a)

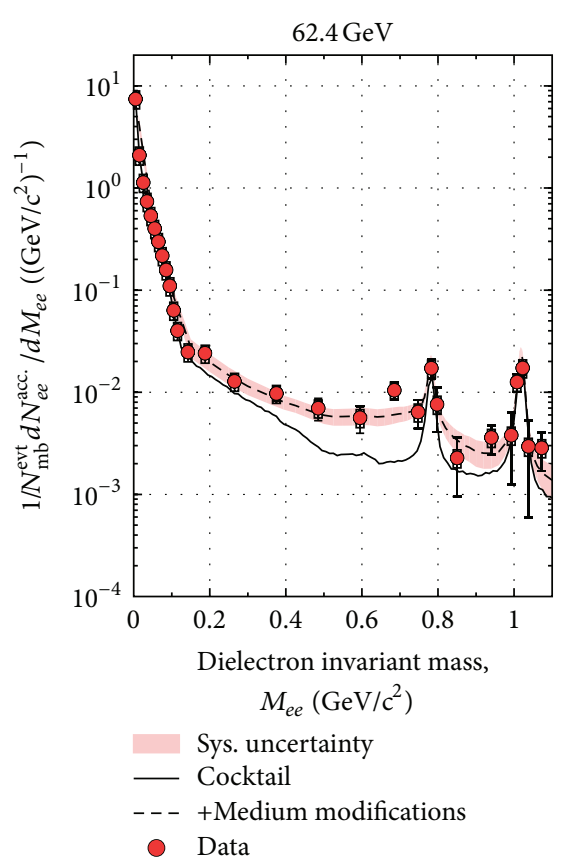

(b)

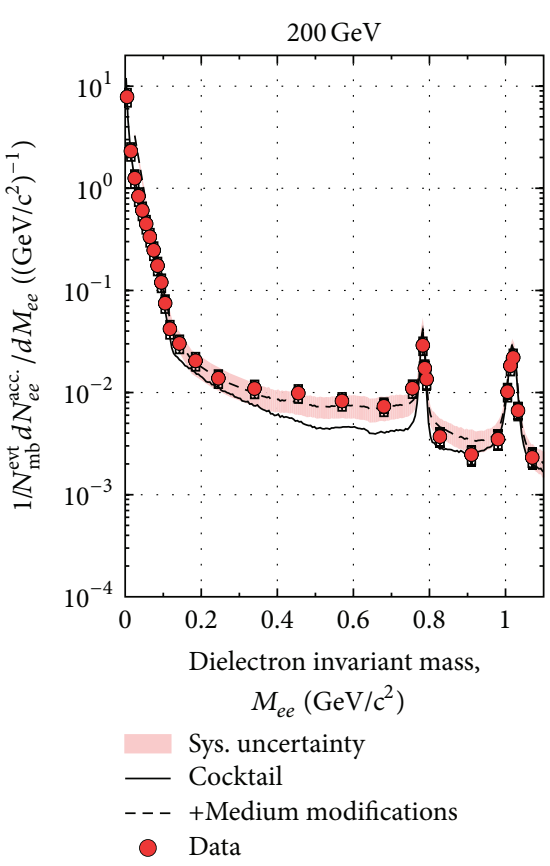

(c)

FIGURE 9: Low-mass dilepton spectra as measured by STAR in the RHIC beam-energy scan [68]; MB spectra are compared to theoretical predictions for the in-medium hadronic + QGP radiation, added to the cocktail contribution.

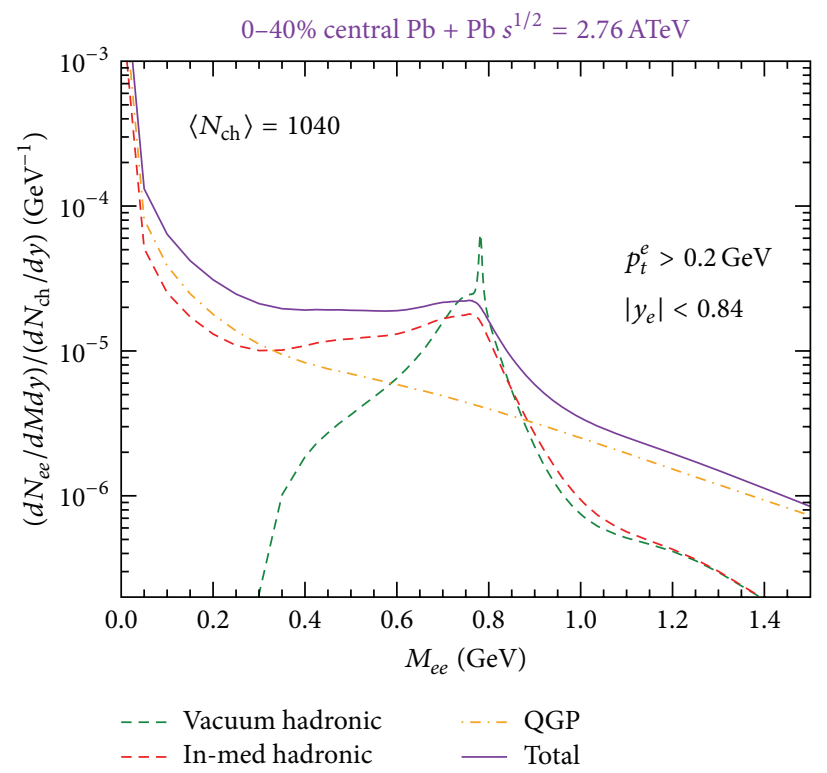

(a)

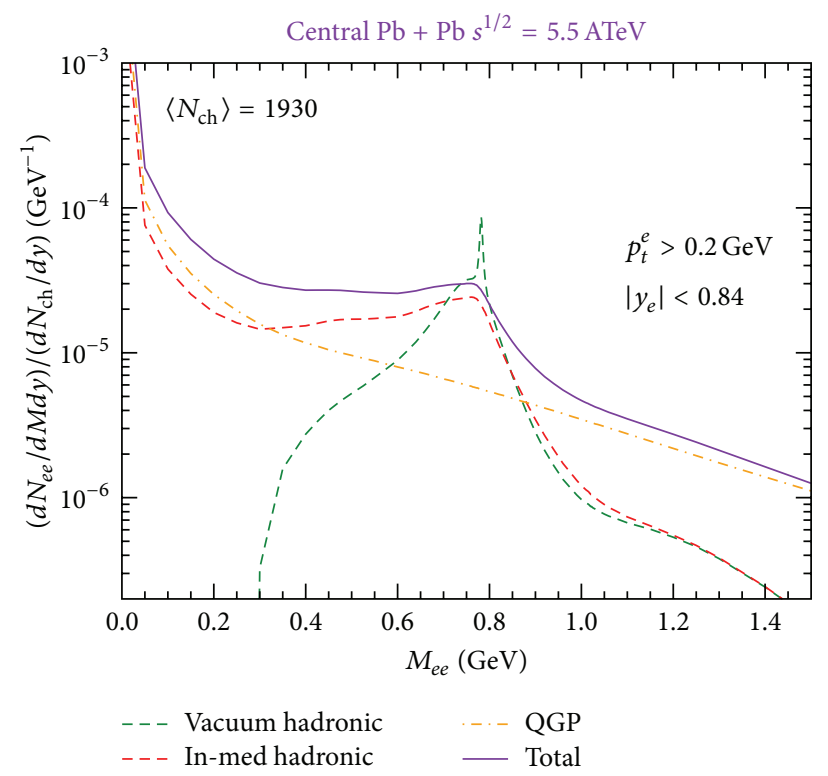

(b)

FIGURE 10: Dielectron invariant-mass spectra from thermal radiation in $0-40 \%$ central $\mathrm{Pb}-\mathrm{Pb}(2.76 \mathrm{ATeV})$ (a) and $0-10 \%$ central $\mathrm{Pb}$ $\mathrm{Pb}(5.5 \mathrm{ATeV})(\mathrm{b})$, including single-electron cuts to simulate the ALICE acceptance. Hadronic (with in-medium or vacuum EM spectral function) and QGP contributions are shown separately along with the sum of in-medium hadronic plus QGP. Here and in the following LHC plots, both vacuum and in-medium hadronic emission rates in the LMR have been supplemented with the vacuum spectral function in the LMR; that is, no in-medium effects due to chiral mixing have been included (for all RHIC calculations shown in the previous sections, full chiral mixing was included). 


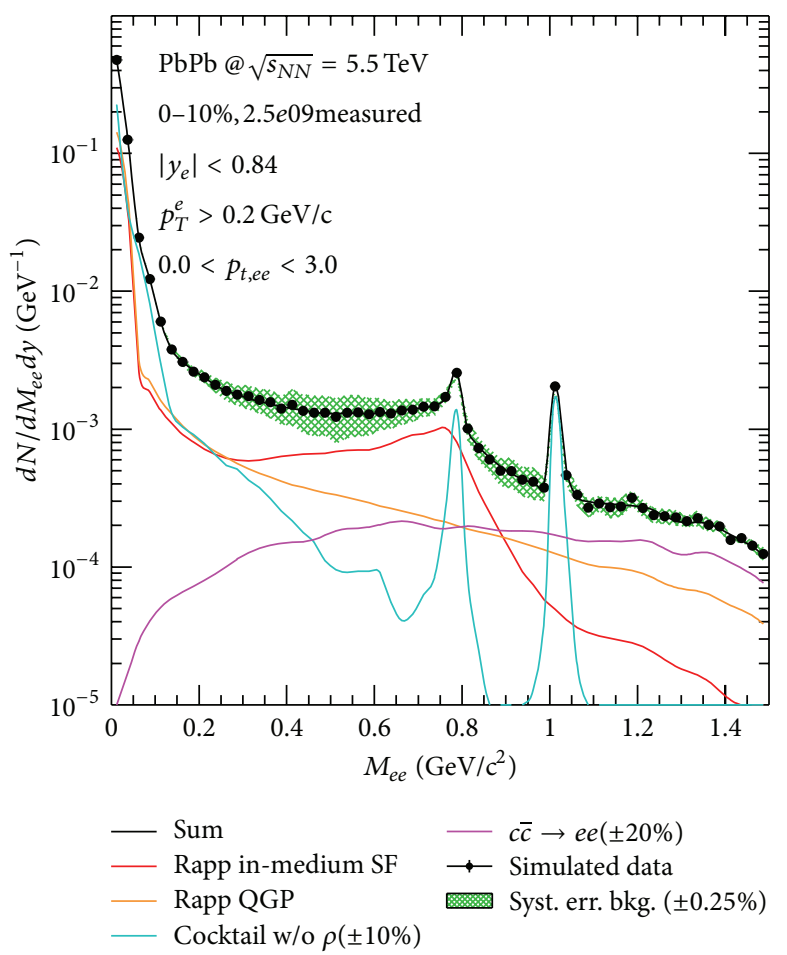

(a)

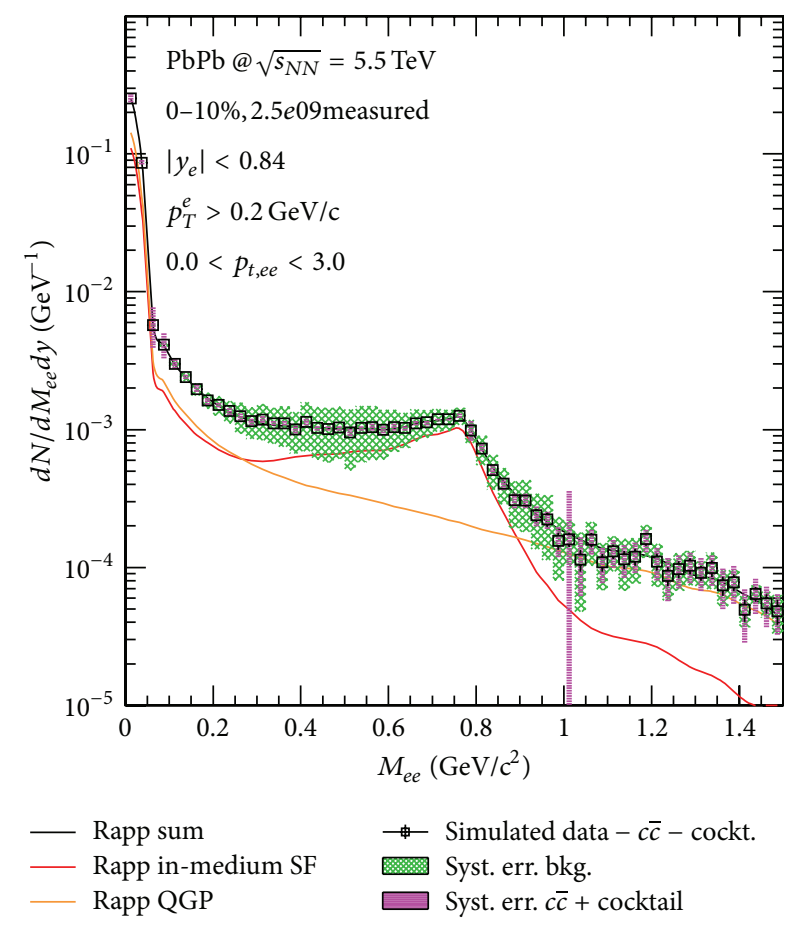

(b)

Figure 11: Simulations of dielectron invariant-mass spectra in $\mathrm{Pb}-\mathrm{Pb}(5.5 \mathrm{ATeV})$ collisions assuming the thermal spectra shown in (a) of Figure 10 as the excess signal $[83,84]$. In addition to the acceptance cuts on single-electron rapidity and momentum, pair efficiency and displaced vertex cuts are included here. (a) Invariant-mass spectra after subtraction of combinatorial background; in addition to the thermal signal, the simulated data contain the hadronic cocktail and correlated open-charm decays. (b) Simulated excess spectra after subtraction of the cocktail and the open-charm contribution using displaced vertex cuts.

function in the LMR which are critical for being able to evaluate signatures of chiral restoration (as discussed elsewhere, see, e.g., $[17,85])$. In addition, the yield and spectral slope of the dominantly QGP emission in the IMR will open a pristine window on QGP lifetime and temperature (recall that the $M$ spectra, which are little affected by the acceptance cuts in the IMR, are unaffected by blue shifts).

Let us turn to the dilepton $q_{t}$ spectra at full LHC energy, displayed again for two mass bins representing the LMR and IMR in Figure 12. Compared to RHIC, the LHC fireball is characterized by a marked increase in QGP lifetime and associated build-up of transverse flow by the time the system has cooled down to $T_{\mathrm{pc}}$. Consequently, the $q_{t}$ spectra exhibit an appreciable increase in their inverse-slope parameters, by about 60\% in the LMR (for both hadronic and QGP parts) and for the QGP part in the IMR, and up to $80 \%$ for the hadronic IMR radiation (recall that in a scenario with chiral mixing, the hadronic radiation for $M=1.1-1.5 \mathrm{GeV}$ is expected to increase by about a factor of 2 , so that its larger slope compared to the QGP will become more significant for the total).

3.5.2. Dimuons. Low-mass dilepton measurements are also possible with ALICE in the dimuon channel at forward rapidities, $2.5<y_{\mu}<4$, albeit with somewhat more restrictive momentum cuts [86]. The charged-particle multiplicity

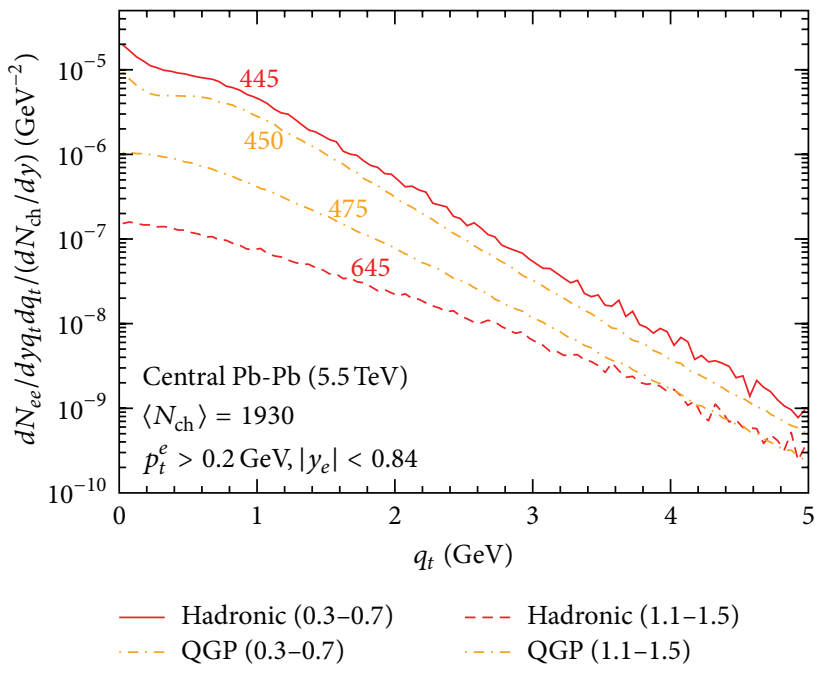

Figure 12: Same as Figure 6, but for central $\mathrm{Pb}-\mathrm{Pb}(5.5 \mathrm{ATeV})$.

in this rapidity range is reduced by about $30 \%$ compared to midrapidity [87] but, at $2.76 \mathrm{ATeV}$, is still ca. $30 \%$ above central rapidities in central $\mathrm{Au}-\mathrm{Au}$ at RHIC.

Figure 13 illustrates the expected thermal mass spectra in central $\mathrm{Pb}-\mathrm{Pb}(2.76 \mathrm{ATeV})$. For "conservative" cuts on 


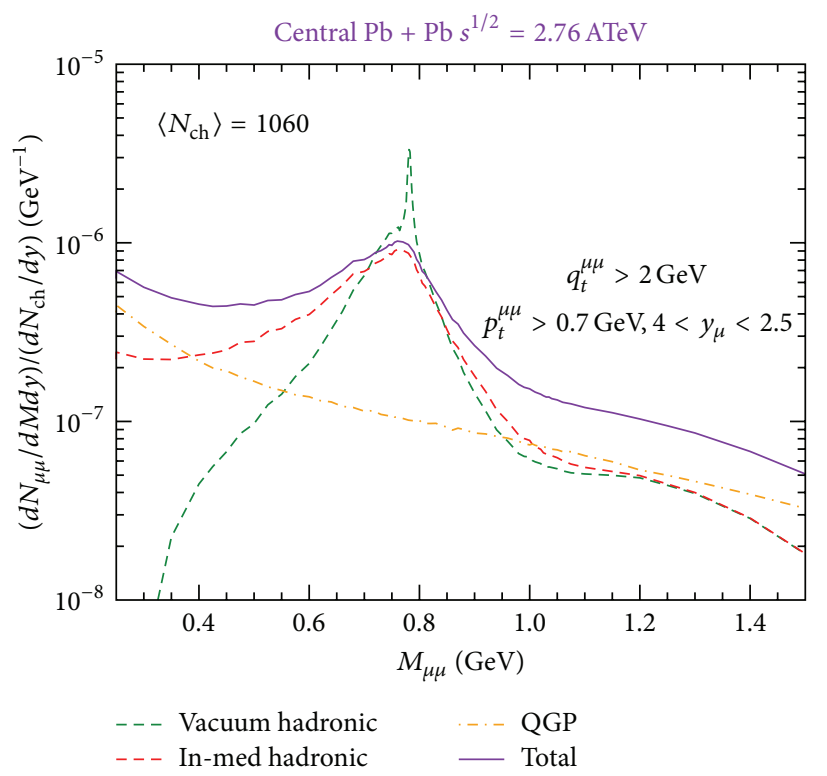

(a)

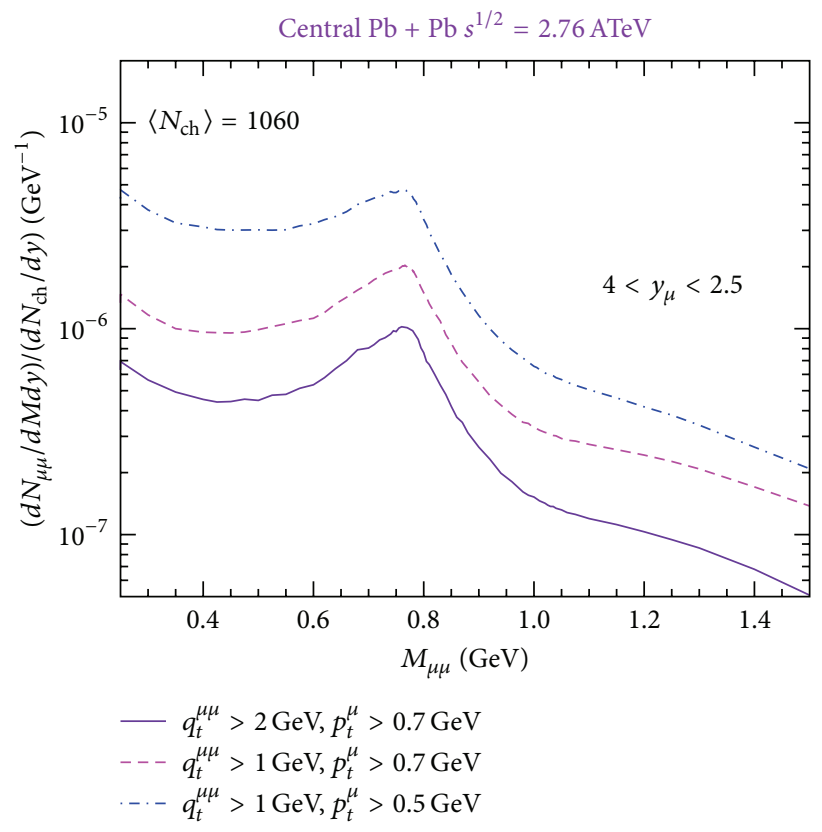

(b)

Figure 13: Calculations of thermal dimuon invariant-mass spectra in central $\mathrm{Pb}-\mathrm{Pb}(2.76 \mathrm{ATeV})$ collisions at forward rapidity, $y=2.5-4$. (a) in-medium hadronic, vacuum hadronic, QGP and the sum of in-medium hadronic plus QGP, are shown with "strong" cuts on single and dimuon transverse momenta. Part (b) illustrates how the total yield increases when the two cuts are relaxed.

the di-/muons $\left(q_{t}^{\mu \mu}>2 \mathrm{GeV}, p_{t}^{\mu}>0.7 \mathrm{GeV}\right)$, their yield is substantially suppressed (see Figure 13(a)), by about one order of magnitude, compared to a typical single-e cut of $p_{t}^{e}>0.2 \mathrm{GeV}$. In addition, the spectral broadening of the inmedium $\rho$ meson is less pronounced, a trend that was also observed in the $q_{t}$-sliced NA60 dimuon spectra. Here, it is mostly due to the suppression of medium effects at larger $\rho$-meson momentum relative to the heat bath, caused by hadronic form factors (analogous to RHIC, recall Figure 7). It is, in fact, mostly the pair cut which is responsible for the suppression, since $q_{t}^{\mu \mu \text {,cut }}$ is significantly larger than $2 p_{t}^{\mu \text {,cut }}$. If the former can be lowered to, say, $1 \mathrm{GeV}$, the thermal yield of accepted pairs increases by about a factor 3 in the IMR and 2 in the LMR (see dashed line in Figure 13(b)). The LMR acceptance is now mainly limited by the single- $\mu$ cut, as the latter suppresses low-mass pairs whose pair momentum is not at least $2 p_{t}^{\mu, \text { cut }}$ (the same effect is responsible for the rather sharp decrease in acceptance for low-momentum electron pairs below $M \simeq 0.4 \mathrm{GeV}$ in Figure $7(\mathrm{a})$, leading to a dip toward lower mass in the thermal spectra, even though the thermal rate increases approximately exponentially). This could be much improved by lowering the single- $\mu$ cut to, for example, $0.5 \mathrm{GeV}$, which would increase the low-mass yield by about a factor of 3. At the same time, the spectral broadening of the $\rho$ becomes more pronounced in the accepted yields; that is, the data would be more sensitive to medium effects.

\section{Summary and Outlook}

In this article, we have given an overview of medium modifications of the electromagnetic spectral function under conditions expected at collider energies (high temperature and small baryon chemical potential) and how these medium effects manifest themselves in experimental dilepton spectra at RHIC and LHC. For the emission rates from the hadronic phase, we have focused on predictions from effective hadronic Lagrangians evaluated with standard manybody (or thermal field-theory) techniques; no in-medium changes of the parameters in the Lagrangian (masses and couplings) have been assumed. Since this approach turned out to describe dilepton data at the SPS well, providing testable predictions for upcoming measurements at RHIC and LHC is in order. As collision energy increases, the QGP occupies an increasing space-time volume of the fireball evolution. To improve the description of the pertinent dilepton radiation, information from lattice-QCD has been implemented on (i) the equation of state around and above $T_{\mathrm{pc}}$ and (ii) nonperturbative dilepton emission rates in the QGP. The latter have been "minimally" extended to finite 3-momentum to facilitate their use in calculations of observables. Since these rates are rather similar to previously employed perturbative (HTL) rates, an approximate degeneracy of the in-medium hadronic and the lQCD rates close to $T_{\mathrm{pc}}$ still holds. This is welcome in view of the smooth crossover transition as deduced from bulk properties and order parameters at $\mu_{q}=0$.

The main features of the calculated thermal spectra at RHIC and LHC are as follows. The crossover in the lQCD EoS produces a noticeable increase of the QGP fraction of the yields (compared to a bag-model EoS), while the hadronic portion decreases (its former mixed-phase contribution has been swallowed by the QGP). However, due to the neardegeneracy of the QGP and hadronic emission rates near 
$T_{\mathrm{pc}}$, both the total yield and its spectral shape change little; the hadronic part remains prevalent in an extended region around the $\rho$ mass at all collision energies. The very fact that an appreciable reshuffling of hadronic and QGP contributions from the transition region occurs indicates that the latter is a dominant source of low-mass dileptons at both RHIC and LHC. This creates a favorable setup for in-depth studies of the chiral restoration transition in a regime of the phase diagram where quantitative support from lQCD computations for order parameters and the EM correlator is available. Current ideas of how to render these connections rigorous have been reported elsewhere. Phenomenologically, it turns out that current RHIC data for LMR dilepton spectra are consistent with the melting $\rho$ scenario (with the caveat of the central $\mathrm{Au}-\mathrm{Au}$ PHENIX data), including a recent first measurement of an excitation function all the way down to SPS energies. However, the accuracy of the current data does not yet suffice to discriminate in-medium spectral functions which differ considerably in detail. These "details" will have to be ironed out to enable definite tests of chiral restoration through the EM spectral function.

While the low-mass shape of the spectra is expected to be remarkably stable with collision energy, large variations are predicted in the excitation function of other dilepton observables. First, the total yields increase substantially with collision energy. In the LMR, the dependence on chargedparticle rapidity density, $N_{\mathrm{ch}}^{\alpha}$, is estimated to scale as $\alpha_{E} \simeq 1.8$ from RHIC to LHC, significantly stronger than as function of centrality at fixed $\sqrt{s}$. This is, of course, a direct consequence of the longer time it takes for the fireball to cool down to thermal freezeout. For the RHIC beam-energy scan program, this opens an exciting possibility to search for a nonmonotonous behavior in the fireball's lifetime due to a critical slowing down of the system's expansion. If the LMR radiation indeed emanates largely from the transition region, a slowed expansion around $T_{c}$ would take maximal advantage of this, thus rendering an "anomalous dilepton enhancement" a promising signature of a critical point.

Second, the transverse-momentum spectra of thermal dileptons are expected to become much harder with collision energy, reflecting the increase in the collective expansion generated by the QGP prior to the transition region. This "QGP barometer" provides a higher sensitivity than finalstate hadron spectra which include the full collectivity of the hadronic evolution. The inverse-slope parameters for $q_{t}$ spectra in the LMR are expected to increase from $\sim 220 \mathrm{MeV}$ at SPS to $\sim 280 \mathrm{MeV}$ at RHIC-200 and up to $\sim 450 \mathrm{MeV}$ at LHC5500. Even larger values are reached in the IMR, although the situation is a bit more involved here, since (a) the QGP emission is increasingly emitted from earlier phases and (b) the hadronic emission, while picking up the full effect of additional collectivity at $T_{\mathrm{pc}}$, becomes subleading relative to the QGP. The trend in the LMR seems to line up with the recent slope measurements in photon excess spectra at RHIC and LHC. A similar connection exists for the elliptic flow; pertinent data will be of great interest. Invariant-mass spectra in the IMR remain the most promising observable to measure early QGP temperatures, once the correlated heavy-flavor decays can be either subtracted or reliably evaluated theoretically.

The versatility of dileptons at collider energies comprises a broad range of topics, ranging from chiral restoration to direct-temperature measurements, QGP collectivity, and fireball lifetime. Experimental efforts are well underway to exploit these, while sustained theoretical efforts will be required to provide thorough interpretations.

\section{Acknowledgments}

The author gratefully acknowledges the contributions of his collaborators, in particular Charles Gale, Min He, and Hendrik van Hees. He also thanks the STAR and ALICE collaborations for making available their plots shown in this article. This work has been supported by the U.S. National Science Foundation under Grant nos. PHY-0969394 and PHY1306359 and by the A.-v.-Humboldt Foundation (Germany).

\section{References}

[1] E. Shuryak, "Physics of strongly coupled quark-gluon plasma," Progress in Particle and Nuclear Physics, vol. 62, no. 1, pp. 48-101, 2009.

[2] B. Friman, C. Höhne, J. Knoll et al., Eds., The CBM Physics Book: Compressed Baryonic Matter in Laboratory Experiments, vol. 814 of Lecture Notes in Physics, 2011.

[3] S. Borsanyi, Z. Fodor, C. Hoelbling et al., "QCD equation of state at nonzero chemical potential: continuum results with physical quark masses at order $\mu^{2}$," Journal of High Energy Physics, vol. 1009, article 073, 2010.

[4] A. Bazavov, T. Bhattacharya, M. Cheng et al., "Chiral and deconfinement aspects of the QCD transition," Physical Review D, vol. 85, no. 5, Article ID 054503, 2012.

[5] F. Weber, "Strange quark matter and compact stars," Progress in Particle and Nuclear Physics, vol. 54, no. 1, pp. 193-288, 2005.

[6] Y. Schutz and U. A. Wiedemann, Eds., "Proceedings of XXII International Conference on Ultrarelativistic Nucleus-Nucleus Collisions, Annecy (France, May 23-28, 2011)," Journal of Physics G, vol. 38, 2012.

[7] T. Ulrich, B. Wyslouch, and J. W. Harris, Eds., "Proceedings of XXIII International Conference on Ultrarelativistic Nucleus-Nucleus Collisions, Washington (DC, Aug. 12-18, 2012)," Nuclear Physics A, vol. 904-905, 2013.

[8] I. Tserruya, "Electromagnetic probes," in Relativistic Heavy-Ion Physics, R. Stock and L. Börnstein, Eds., New Series I/23A, Springer, 2010.

[9] H. J. Specht, “Thermal dileptons from hot and dense strongly interacting matter," in Proceedings of International Workshop on Chiral Symmetry in Hadrons and Nuclei, vol. 1322 of AIP Conference Proceedings, pp. 1-10, Valencia, Spain, June 2010.

[10] R. Rapp, J. Wambach, and H. van Hees, "The chiral restoration transition of QCD and low mass dileptons," in Relativistic Heavy-Ion Physics, R. Stock and L. Börnstein, Eds., New Series I/23A, Springer, 2010.

[11] P. Braun-Munzinger and J. Stachel, "Hadron production in ultra-relativistic nuclear collisions and the QCD phase diagram: an update," in From Nuclei to Stars: Festschrift in Honor of Gerald E Brown's 85th Birthday, S. Lee, Ed., World Scientific, Singapore, 2011. 
[12] R. Rapp, "Hadrochemistry and evolution of (anti)baryon densities in ultrarelativistic heavy-ion collisions," Physical Review C, vol. 66, no. 1, Article ID 017901, 4 pages, 2002.

[13] R. Rapp, "Signatures of thermal dilepton radiation at ultrarelativistic energies," Physical Review C, vol. 63, no. 5, Article ID 054907, 13 pages, 2001.

[14] H.-T. Ding, A. Francis, O. Kaczmarek, F. Karsch, E. Laermann, and W. Soeldner, "Thermal dilepton rate and electrical conductivity: an analysis of vector current correlation functions in quenched lattice QCD," Physical Review D, vol. 83, no. 3, Article ID 034504, 2011.

[15] B. B. Brandt, A. Francis, H. B. Meyer, and H. Wittig, “Thermal correlators in the $\rho$ channel of two-flavor QCD," Journal of High Energy Physics, vol. 1303, article 100, 2013.

[16] Y. Aoki, G. Endrodi, Z. Fodor, S. D. Katz, and K. K. Szabó, “The order of the quantum chromodynamics transition predicted by the standard model of particle physics," Nature, vol. 443, no. 7112, pp. 675-678, 2006.

[17] R. Rapp, "Update on chiral symmetry restoration in the context of dilepton data," Journal of Physics: Conference Series, vol. 420, Article ID 012017, 2013.

[18] S. Leupold, V. Metag, and U. Mosel, "Hadrons in strongly interacting matter," International Journal of Modern Physics E, vol. 19, no. 2, pp. 147-224, 2010.

[19] E. Oset, A. Ramos, E. J. Garzon et al., "Interaction of vector mesons with baryons and nuclei," International Journal of Modern Physics E, vol. 21, no. 11, Article ID 1230011, 2012.

[20] M. Dey, V. L. Eletsky, and B. L. Ioffe, "Mixing of vector and axial mesons at finite temperature: an indication towards chiral symmetry restoration," Physics Letters B, vol. 252, no. 4, pp. 620624, 1990.

[21] V. L. Eletsky, M. Belkacem, P. J. Ellis, and J. I. Kapusta, "Properties of $\rho$ and $\omega$ mesons at finite temperature and density as inferred from experiment," Physical Review C, vol. 64, no. 3, Article ID 035202, 2001.

[22] M. Harada and K. Yamawaki, "Hidden local symmetry at loop: a new perspective of composite gauge boson and chiral phase transition," Physics Reports, vol. 381, no. 1-3, pp. 1-233, 2003.

[23] B. Friman and H. J. Pirner, "P-wave polarization of the $\rho$-meson and the dilepton spectrum in dense matter," Nuclear Physics A, vol. 617, no. 4, pp. 496-509, 1997.

[24] R. Rapp, G. Chanfray, and J. Wambach, "Rho meson propagation and dilepton enhancement in hot hadronic matter," Nuclear Physics A, vol. 617, no. 4, pp. 472-495, 1997.

[25] S. Ghosh and S. Sarkar, " $\rho$ self-energy at finite temperature and density in the real-time formalism," Nuclear Physics A, vol. 870871, pp. 94-111, 2011, Erratum in Nuclear Physics A, vol. 888, p. 44, 2012.

[26] J. Cleymans, J. Fingberg, and K. Redlich, “Transverse-momentum distribution of dileptons in different scenarios for the QCD phase transition," Physical Review D, vol. 35, no. 7, pp. 2153-2165, 1987.

[27] E. Braaten, R. D. Pisarski, and T. C. Yuan, "Production of soft dileptons in the quark-gluon plasma," Physical Review Letters, vol. 64, no. 19, pp. 2242-2245, 1990.

[28] O. Kaczmarek, E. Laermann, M. Mueller et al., "Thermal dilepton rates from quenched lattice QCD," PoS Confinement $X$, vol. 2012, pp. 185-192, 2012.

[29] G. D. Moore and J.-M. Robert, "Dileptons, spectral weights, and conductivity in the quark-gluon plasma," http://arxiv.org/abs/ hep-ph/0607172.
[30] J. Kapusta, P. Lichard, and D. Seibert, "High-energy photons from quark-gluon plasma versus hot hadronic gas," Physical Review D, vol. 44, no. 9, pp. 2774-2788, 1991, Erratum in Physical Review D, vol. 47, no. 9, p. 4171, 1993.

[31] P. B. Arnold, G. D. Moore, and L. G. Yaffe, "Photon emission from quark-gluon plasma: complete leading order results," Journal of High Energy Physics, vol. 2001, no. 12, article 009, 2001.

[32] G. Aarts, C. Allton, J. Foley, S. Hands, and S. Kim, "Spectral functions at small energies and the electrical conductivity in hot quenched lattice QCD," Physical Review Letters, vol. 99, no. 2, Article ID 022002, 4 pages, 2007.

[33] K. Nakamura, "Review of particle physics," Journal of Physics G, vol. 37, no. 7A, Article ID 075021, 2010.

[34] R. Rapp and J. Wambach, "Chiral symmetry restoration and dileptons in relativistic heavy-ion collisions," in Advances in Nuclear Physics, J. W. Negele and E. Vogt, Eds., vol. 25, pp. 1205, 2002.

[35] R. Rapp and J. Wambach, "Low-mass dileptons at the CERNSpS: evidence for chiral restoration?" The European Physical Journal A, vol. 6, no. 4, pp. 415-420, 1999.

[36] M. Urban, M. Buballa, R. Rapp, and J. Wambach, "Momentum dependence of the pion cloud for $\rho$-mesons in nuclear matter," Nuclear Physics A, vol. 641, no. 4, pp. 433-460, 1998.

[37] R. Rapp, "Hadrons in hot and dense matter," European Physical Journal A, vol. 18, no. 2-3, pp. 459-462, 2003.

[38] U. W. Heinz and R. Snellings, "Collective flow and viscosity in relativistic heavy-ion collisions," Annual Review of Nuclear and Particle Science, vol. 63, 2013.

[39] P. F. Kolb and U. W. Heinz, "Hydrodynamic description of ultrarelativistic heavy-ion collisions," in Quark Gluon Plasma* 634-714, R. C. Hwa and X.-N. Wang, Eds., World Scientific, Singapore, 2004.

[40] S. Borsanyi, G. Endrodi, Z. Fodor et al., "The QCD equation of state with dynamical quarks," Journal of High Energy Physics, vol. 2010, no. 5, article 077, 2010.

[41] M. Cheng, S. Ejiri, P. Hegde et al., "Equation of state for physical quark masses," Physical Review D, vol. 81, no. 5, Article ID 054504, 2010.

[42] M. He, R. J. Fries, and R. Rapp, "Ideal hydrodynamics for bulk and multistrange hadrons in $\sqrt{s_{N N}}=200 \mathrm{~A} \mathrm{GeV} \mathrm{Au-Au} \mathrm{colli-}$ sions," Physical Review C, vol. 85, no. 4, Article ID 044911, 9 pages, 2012.

[43] S. Pratt, "Resolving the hanbury brown-twiss puzzle in relativistic heavy ion ollisions," Physical Review Letters, vol. 102, no. 23, Article ID 232301, 4 pages, 2009.

[44] T. Renk, R. Schneider, and W. Weise, "Phases of QCD, thermal quasiparticles, and dilepton radiation from a fireball," Physical Review C, vol. 66, no. 1, Article ID 014902, 19 pages, 2002.

[45] H. van Hees and R. Rapp, "Dilepton radiation at the CERN super-proton synchrotron," Nuclear Physics A, vol. 806, no. 14, pp. 339-387, 2008.

[46] H. van Hees et al., In preparation.

[47] S. Turbide, C. Gale, D. K. Srivastava, and R. J. Fries, "High momentum dilepton production from jets in a quark gluon plasma," Physical Review C, vol. 74, no. 1, Article ID 014903, 2006.

[48] A. Adare, S. Afanasiev, C. Aidala et al., "Detailed measurement of the $e^{+} e^{-}$pair continuum in $p+p$ and $\mathrm{Au}+\mathrm{Au}$ collisions at $\sqrt{s_{N N}}=200 \mathrm{GeV}$ and implications for direct photon production," Physical Review C, vol. 81, no. 3, Article ID 034911, 56 pages, 2010. 
[49] J. Zhao, "Dielectron continuum production from $\sqrt{\mathrm{s}_{N N}}=$ $200 \mathrm{GeV} p+p$ and $\mathrm{Au}+\mathrm{Au}$ collisions at STAR," Journal of Physics G, vol. 38, no. 12, Article ID 124134, 2011.

[50] J. Zhao, Private Communication, 2012.

[51] G. Vujanovic, C. Young, B. Schenke, S. Jeon, R. Rapp, and C. Gale, "Dilepton production in high energy heavy ion collisions with $3+1 \mathrm{D}$ relativistic viscous hydrodynamics," Nuclear Physics A, vol. 904-905, pp. 557c-560c, 2013.

[52] H.-J. Xu, H.-F. Chen, X. Dong, Q. Wang, and Y.-F. Zhang, "Di-electron production from vector mesons with medium modifications in heavy ion collisions," Physical Review C, vol. 85, no. 2, Article ID 024906, 8 pages, 2012.

[53] O. Linnyk, W. Cassing, J. Manninen, E. L. Bratkovskaya, and C. M. Ko, "Analysis of dilepton production in $\mathrm{Au}+\mathrm{Au}$ collisions at $\sqrt{s_{N N}}=200 \mathrm{GeV}$ within the parton-hadron-string dynamics transport approach," Physical Review C, vol. 85, no. 2, Article ID 024910, 2012.

[54] I. Tserruya, "Photons and low-mass dileptons: results from PHENIX," Nuclear Physics A, vol. 904-905, pp. 225c-232c, 2013.

[55] K. Dusling and I. Zahed, "Low mass dilepton radiation at RHIC," Nuclear Physics A, vol. 825, no. 3-4, pp. 212-221, 2009.

[56] S. Ghosh, S. Sarkar, and J.-E. Alam, "Observing many-body effects on lepton pair production from low mass enhancement and flow at RHIC and LHC energies," The European Physical Journal C, vol. 71, article 1760, 2011.

[57] M. Chiu, T. K. Hemmick, V. Khachatryan, A. Leonidov, J. Liao, and L. McLerran, "Production of photons and dileptons in the glasma," Nuclear Physics A, vol. 900, pp. 16-37, 2013.

[58] S. Turbide, R. Rapp, and C. Gale, "Hadronic production of thermal photons," Physical Review C, vol. 69, no. 1, Article ID 014903, 13 pages, 2004.

[59] P. J. Siemens and S. A. Chin, "Testing of QCD plasma formation by dilepton spectra in relativistic nuclear collisions," Physical Review Letters, vol. 55, no. 12, pp. 1266-1268, 1985.

[60] K. Kajantie, J. Kapusta, L. McLerran, and A. Mekjian, "Dilepton emission and the QCD phase transition in ultrarelativistic nuclear collisions," Physical Review D, vol. 34, no. 9, pp. 27462754, 1986.

[61] B. Kämpfer and O. P. Pavlenko, "Probing meson spectral functions with double differential dilepton spectra in heavy-ion collisions," The European Physical Journal A, vol. 10, no. 1, pp. 101-107, 2001.

[62] T. Renk and J. Ruppert, "Dimuon transverse momentum spectra as a tool to characterize the emission region in heavy-ion collisions," Physical Review C, vol. 77, no. 2, Article ID 024907, 7 pages, 2008.

[63] J. K. Nayak, J.-E. Alam, T. Hirano, S. Sarkar, and B. Sinha, "Muon pairs from In + In collision at energies available at the CERN super proton synchrotron," Physical Review C, vol. 85, no. 6, Article ID 064906, 6 pages, 2012.

[64] J. Deng, Q. Wang, N. Xu, and P. Zhuang, "Dilepton flow and deconfinement phase transition in heavy ion collisions," Physics Letters B, vol. 701, no. 5, pp. 581-586, 2011.

[65] R. Arnaldi, K. Banicz, K. Borer et al., "NA60 results on thermal dimuons," The European Physical Journal C, vol. 61, no. 4, pp. 711-720, 2009.

[66] G. Agakichiev, H. Appelshauser, J. Bielcikova et al., " $e^{+} e^{-}$-pair production in $\mathrm{Pb}-\mathrm{Au}$ collisions at $158 \mathrm{GeV}$ per nucleon," The European Physical Journal C, vol. 41, no. 4, pp. 475-513, 2005.

[67] M. He, R. J. Fries, and R. Rapp, In preparation.
[68] F. Geurts, “The STAR dilepton physics program," Nuclear Physics A, vol. 904-905, pp. 217c-224c, 2013.

[69] R. Chatterjee, D. K. Srivastava, U. Heinz, and C. Gale, "Elliptic flow of thermal dileptons in relativistic nuclear collisions," Physical Review C, vol. 75, no. 5, Article ID 054909, 2007.

[70] P. Mohanty, V. Roy, S. Ghosh et al., "Elliptic flow of thermal dileptons as a probe of QCD matter," Physical Review C, vol. 85, no. 3, Article ID 031903, 2012.

[71] A. Adare, S. Afanasiev, C. Aidala et al., "Enhanced production of direct photons in $\mathrm{Au}+\mathrm{Au}$ collisions at $\sqrt{\mathrm{s}_{N N}}=200 \mathrm{GeV}$ and implications for the initial temperature," Physical Review Letters, vol. 104, no. 13, Article ID 132301, 6 pages, 2010.

[72] A. Adare, S. Afanasiev, C. Aidala et al., "Observation of direct-photon collective flow in $\mathrm{Au}+\mathrm{Au}$ collisions at $\sqrt{\mathrm{s}_{N N}}=$ 200 GeV," Physical Review Letters, vol. 109, no. 12, Article ID 122302, 7 pages, 2012.

[73] M. Wilde, A. Deloff, I. Ilkiv et al., "Measurement of direct photons in $p+p$ and $\mathrm{Pb}-\mathrm{Pb}$ collisions with ALICE," Nuclear Physics A, vol. 904-905, pp. 573c-576c, 2013.

[74] D. Lohner, "Measurement of direct-photon elliptic flow in $\mathrm{Pb}$ $\mathrm{Pb}$ collisions at $\sqrt{\mathrm{s}_{N N}}=2.76 \mathrm{TeV}$," http://arxiv.org/abs/1212.3995.

[75] H. van Hees, C. Gale, and R. Rapp, “Thermal photons and collective flow at energies available at the BNL relativistic heavyion collider," Physical Review C, vol. 84, no. 5, Article ID 054906, 2011.

[76] F. M. Liu, T. Hirano, K. Werner, and Y. Zhu, "Elliptic flow of thermal photons in $\mathrm{Au}+\mathrm{Au}$ collisions at $\sqrt{s_{N N}}=200 \mathrm{GeV}$," Physical Review C, vol. 80, no. 3, Article ID 034905, 5 pages, 2009.

[77] H. Holopainen, S. Rasanen, and K. J. Eskola, "Elliptic flow of thermal photons in heavy-ion collisions at energies available at the BNL relativistic heavy ion collider and at the CERN large hadron collider," Physical Review C, vol. 84, no. 6, Article ID 064903, 11 pages, 2011.

[78] M. Dion, J.-F. Paquet, B. Schenke, C. Young, S. Jeon, and C. Gale, "Viscous photons in relativistic heavy ion collisions," Physical Review C, vol. 84, no. 6, Article ID 064901, 13 pages, 2011.

[79] G. Başar, D. E. Kharzeev, and V. Skokov, "Conformal anomaly as a source of soft photons in heavy ion collisions," Physical Review Letters, vol. 109, no. 20, Article ID 202303, 5 pages, 2012.

[80] G. Agakichiev, A. Balanda, D. Belver et al., "Origin of the lowmass electron pair excess in light nucleus-nucleus collisions," Physics Letters B, vol. 690, no. 2, pp. 118-122, 2010.

[81] R. Holzmann, “The experimental quest for in-medium effects," in Proceedings of the 6th International Conference on Quarks and Nuclear Physics (QNP '12), vol. 5, Paris, France, April 2012.

[82] D. Adamová, G. Agakichiev, H. Appelshäuser et al., "Enhanced production of low-mass electron-positron pairs in $40-A \mathrm{GeV}$ $\mathrm{Pb}-\mathrm{Au}$ collisions at the CERN SPS," Physical Review Letters, vol. 91, no. 4, Article ID 042301, 5 pages, 2003.

[83] L. Musa and K. Safarik, "Letter of intent for the upgrade of the ALICE experiment," CERN-LHCC-2012-012, CERN, Geneva, Switzerland, 2012, http://cds.cern.ch/record/1475243.

[84] P. Reichelt and H. Appleshäuser, Private Communication, 2013.

[85] P. M. Hohler and R. Rapp, "Evaluating chiral symmetry restoration through the use of sum rules," EPJ Web of Conferences, vol. 36, article 00012, 2012.

[86] R. Tieulent, Private Communication, 2012.

[87] K. Gulbrandsen et al., "Charged-particle pseudorapidity density and anisotropic flow over a wide pseudorapidity range using ALICE at the LHC," in Proceedings of the Hot Quarks Workshop, Copamarina, Puerto Rico, October 2012. 

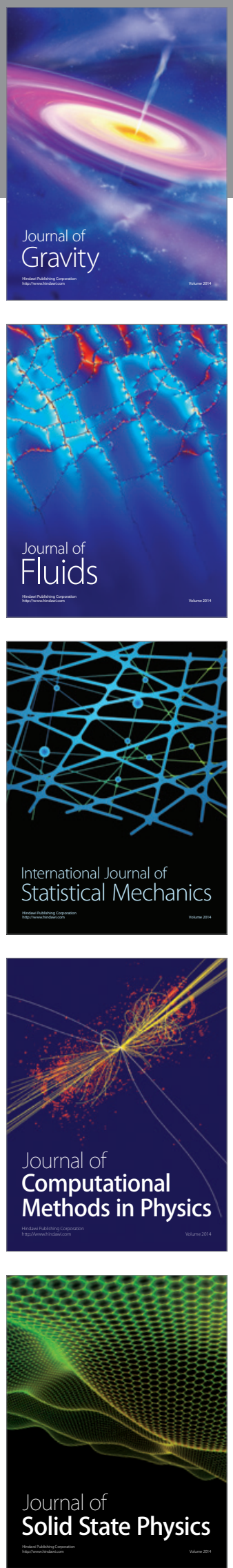

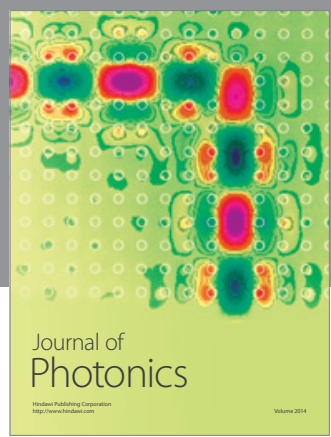

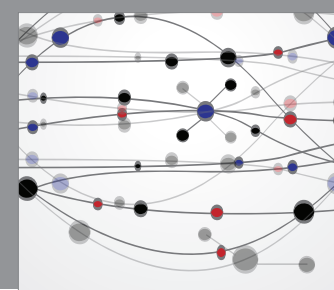

The Scientific World Journal

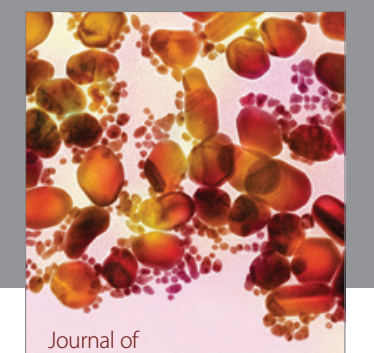

Soft Matter
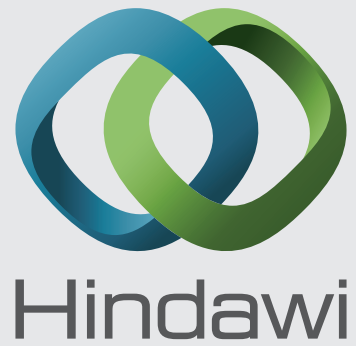

Submit your manuscripts at

http://www.hindawi.com
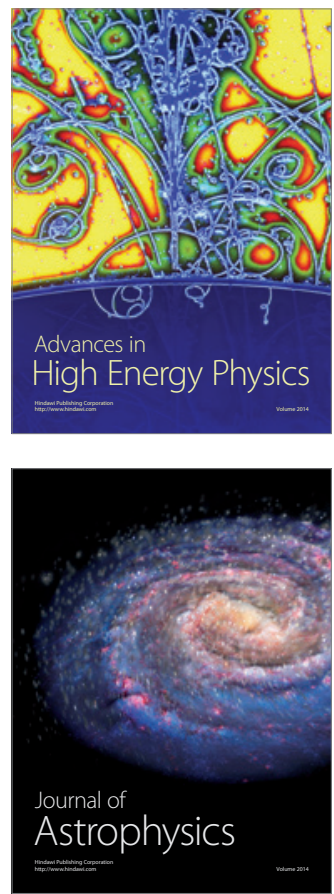
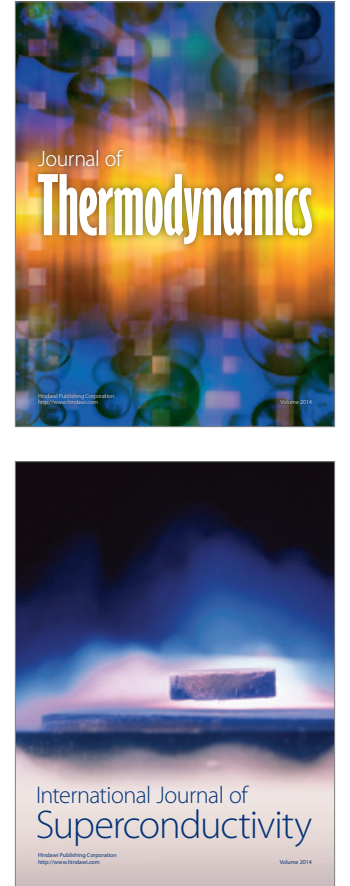
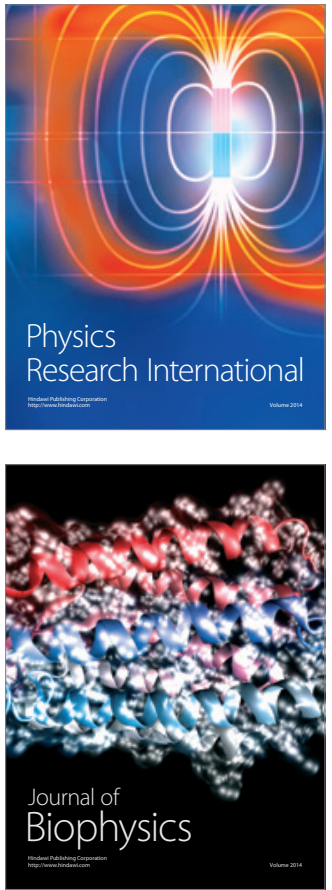
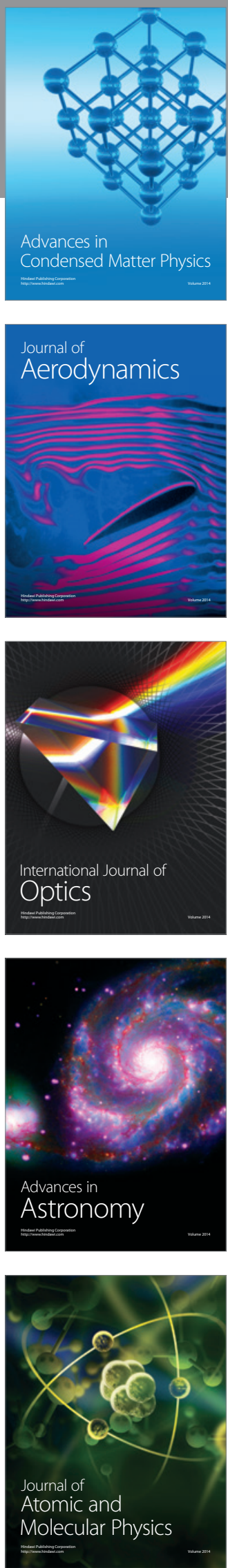